\title{
Astronomy with ultra high-energy particles
}

\author{
Jörg R. Hörandel \\ Department of Astrophysics, Radboud University Nijmegen \\ P.O. Box 9010, 6500 GL Nijmegen, The Netherlands \\ J.Horandel@astro.ru.nl, http://particle.astro.kun.nl
}

\begin{abstract}
Recent measurements of the properties of cosmic rays above $10^{17} \mathrm{eV}$ are summarized and implications on our contemporary understanding of their origin are discussed. Cosmic rays with energies exceeding $10^{20} \mathrm{eV}$ have been measured, they are the highest-energy particles in the Universe. Particles at highest energies are expected to be only marginally deflected by magnetic fields and they should point towards their sources on the sky. Recent results of the Pierre Auger Observatory have opened a new window to the Universe - astronomy with ultra high-energy particles.
\end{abstract}

\section{Introduction}

The Earth is permanently exposed to a vast flux of high-energy particles from outer space. Most of these particles are fully ionized atomic nuclei with relativistic energies. The extraterrestrial origin of these particles has been demonstrated by V. Hess in 1912 [Hess(1912)] and he named the particles "Höhenstrahlung" (high-altitude radiation) or "Ultrastrahlung" (ultra radiation). In 1925 R. Millikan coined the term "Cosmic Rays". They have a threefold origin. Particles with energies below $100 \mathrm{MeV}{ }^{1}$ originate from the Sun Ryan(2005), Kahler et al.(2005)]. Cosmic rays in narrower sense are particles with energies from the $100 \mathrm{MeV}$ domain up to energies beyond $10^{20} \mathrm{eV}$. Up to several $10 \mathrm{GeV}$ the flux of the particles observed is modulated on different time scales by the heliospheric magnetic fields [Fichtner(2005), Heber(2005)]. Particles with energies below $10^{17}$ to $10^{18} \mathrm{eV}$ are usually considered to be of galactic origin [Gaisser and Stanev(2006), Gaisser(2006), Strong et al.(2007), Hörandel(2008), Hörandel(2007a)|. The Larmor radius of a particle with energy $E_{15}$ (in units of $10^{15} \mathrm{eV}$ ) and charge $Z$ in a magnetic field $B_{\mu \mathrm{G}}($ in $\mu \mathrm{G}$ ) is

$$
r_{L}=1.08 \frac{E_{15}}{Z B_{\mu \mathrm{G}}} \mathrm{pc},
$$

yielding a value of $r_{L}=360 \mathrm{pc}$ for a proton with an energy of $10^{18} \mathrm{eV}$ in the galactic magnetic field $\left(B_{\mu \mathrm{G}} \approx 3\right)$. This radius is comparable to the thickness

\footnotetext{
${ }^{1}$ In this review we use the particle physics energy units $\mathrm{MeV}=10^{6} \mathrm{eV}, \mathrm{GeV}=10^{9} \mathrm{eV}$, $\mathrm{TeV}=10^{12} \mathrm{eV}, \mathrm{PeV}=10^{15} \mathrm{eV}$, and $\mathrm{EeV}=10^{18} \mathrm{eV} ; 1 \mathrm{eV}=1.6 \cdot 10^{-19} \mathrm{~J}$.
} 
of the galactic disc and illustrates that particles (at least with small charge $Z$ ) at the highest energies can not be magnetically bound to the Galaxy. Hence, they are considered to be of extragalactic origin [Nagano and Watson(2000), Bergman and Belz(2007), Kampert(2008)].

In the present article, we focus on recent results concerning the origin of the extragalactic particles. Cosmic rays with energies exceeding $10^{20} \mathrm{eV}$ are the highest-energy particles in the Universe. Particles at highest energies are only marginally deflected in the galactic magnetic fields, following (11) they have a Larmor radius $r_{L}>36 \mathrm{kpc}$, exceeding the diameter of the Milky Way. Thus, they should point back to their sources, enabling astronomical observations with charged particles.

Several questions arise, concerning the origin of highest-energy cosmic rays. Among them are:

- What are the energies of the particles? (Sect.4)

- What are these particles? Are they protons, nuclei of heavy atoms like oxygen or iron, furthermore are they photons or neutrinos? (Sect. (5)

- Where do they come from? Can we learn something by studying their arrival directions? (Sect. 6)

- How do they propagate to us? Do they suffer any interactions? (Sect.4)

In the following sections (4) to 6) recent experimental results are compiled and their implications to answer the questions raised above are discussed. Before, possible scenarios for the sources of the particles are summarized (Sect.2.1) and mechanisms are discussed which are important during the propagation of the particles through the Universe (Sect.2.2). The detections methods applied are sketched in Sect.3

\section{Sources and Propagation}

\section{$2.1 \quad$ Sources}

The energy density contained in the flux of extragalactic cosmic rays can be inferred from the measured differential energy spectrum $d N / d E$ Halzen(2006)

$$
\rho_{E}=\frac{4 \pi}{c} \int \frac{E}{\beta} \frac{d N}{d E} d E
$$

where $\beta c$ is the velocity of particles with energy $E$. To estimate the energy content of the extragalactic component, assumptions have to be made about the contribution of galactic cosmic rays at energies in the transition region $\left(10^{17}-10^{18} \mathrm{eV}\right)$. The extragalactic component needed according to the poly-gonato model [Hörandel(2003a)] to sustain the observed all-particle flux at highest energies has an energy density of $\rho_{E}=3.7 \cdot 10^{-7} \mathrm{eV} / \mathrm{cm}^{3}$. The power required for a population of sources to generate this energy density over the Hubble time of $10^{10}$ years is $5.5 \cdot 10^{37} \mathrm{erg} /\left(\mathrm{s} \mathrm{Mpc}^{3}\right)$. This leads to $\approx 2 \cdot 10^{44} \mathrm{erg} / \mathrm{s}$ per active galaxy or $\approx 2 \cdot 10^{52} \mathrm{erg}$ per cosmological gamma ray burst Gaisser(1997)]. The coincidence between these numbers and the 


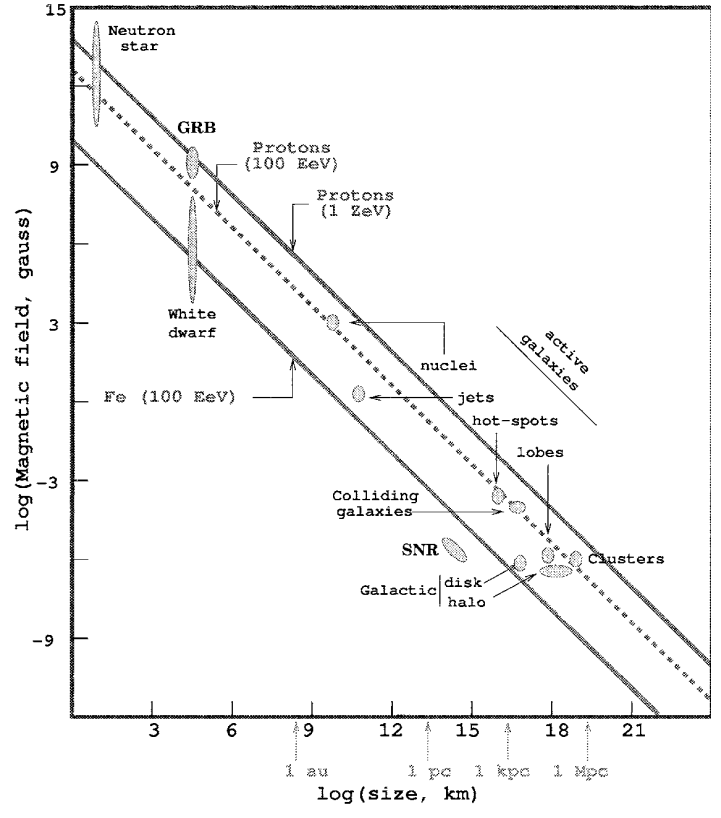

Figure 1: Size and magnetic field strength of possible sites of particle acceleration (Hillas diagram). Acceleration of cosmic rays up to a given energy requires conditions above the respective line [Ostrowski(2002)].

observed output in electromagnetic energy of these sources explains why they are considered as promising candidates to accelerate highest-energy cosmic rays.

The characteristic size of an accelerating region can be estimated for models of gradual acceleration, where the particles make many irregular loops in a magnetic field while gaining energy [Hillas(1984)]. The size $L$ of the essential part of the accelerating region containing the magnetic field must be grater than $2 r_{L}$. A closer look reveals that a characteristic velocity $\beta c$ of scattering centers is of virtual importance [Hillas(1984)], which yields the expression

$$
B_{\mu \mathrm{G}} L_{\mathrm{pc}}>2 E_{15} /(Z \beta) .
$$

It relates the characteristic size $L_{\mathrm{pc}}$ (in pc) and magnetic fields $B_{\mu \mathrm{G}}$ of objects being able to accelerate particles to energies $E_{15}$. Several possible acceleration sites are explored in Fig.1, where the magnetic field strength is plotted as function of their typical sizes [Ostrowski(2002)]. The lines according to (3) represent the conditions for protons and iron nuclei of different energies, as indicated. Objects capable to accelerate particles above a respective energy should be above the respective line. As can be inferred from the figure, most promising candidates to accelerate highestenergy cosmic rays are gamma ray bursts and active galactic nuclei (AGN) [Ginzburg and Syrovatskii(1964), Hillas(1984)]. These objects are typically in a distance of several tens of Mpc to the Earth. Interactions in the source itself or in the vicinity of the source of hadronic particles (protons, nuclei) yield neutral and charged pions, which subsequently decay into high-energy photons and neutrinos. 
Alternatively, so called "top-down models" are discussed in the literature [Hill and Schramm(1983), Olinto(2000), Bhattacharjee and Sigl(2000)]. They have been motivated by events seen by the AGASA experiment above the threshold for the GZK effect [Takeda et al.(1998)]. It is proposed that ultra high-energy particles (instead of being accelerated, "bottom-up scenario") are the decay products of exotic, massive particles originating from highenergy processes in the early Universe. Such super-massive particles (with $m_{X} \gg 10^{11} \mathrm{GeV}$ ) decay e.g. via $\mathrm{W}$ and $\mathrm{Z}$ bosons into high-energy protons, photons, and neutrinos.

\subsection{Propagation}

On the way from their sources to Earth the particles propagate mostly outside galaxies in intergalactic space with very low particle densities. In this environment the most important interactions of cosmic rays occur with photons of the $2.7-\mathrm{K}$ microwave background radiation, namely pair production and pion photoproduction [Hill and Schramm(1985)].

On the last part of their way to Earth they propagate through the Galaxy. However, since particles at the highest energies travel almost along straight lines they accumulate a negligible amount of material during their short travel through regions with relatively high densities. Thus, interactions with the interstellar material can be neglected.

The Universe is filled with about 412 photons $/ \mathrm{cm}^{3}$ of the $2.7^{\circ} \mathrm{K}$ microwave background radiation. Shortly after the discovery of the microwave background it was proposed that ultra high-energy cosmic rays should interact with the photons, leading to a suppression of the observed flux at highest energies Greisen(1966), Zatsepin and Kuz'min(1966)]. This effect is called after its proposers the Greisen-Zatsepin-Kuz'min (GZK) effect. A nucleon of energies exceeding $E_{G Z K} \approx 6 \cdot 10^{19} \mathrm{eV}$ colliding head-on with a $2.7^{\circ} \mathrm{K}$ photon comprises a system of sufficient energy to produce pions by the photoproduction reaction

$$
p+\gamma_{3 K} \rightarrow \Delta^{+} \rightarrow \begin{gathered}
p+\pi^{0} \\
n+\pi^{+}
\end{gathered} .
$$

The energy loss of the nucleon is a significant fraction of the initial energy. The pion photoproduction cross section is quite large above threshold due to resonance production ( $\Delta$ resonance), rising quickly to $500 \mathrm{mb}$ for photon laboratory energies of about $0.3 \mathrm{GeV}$. Subsequent decays of the neutral and charged pions produced in (4) yield high-energy photons and neutrinos.

The center of mass energy of interactions of cosmic rays with energies exceeding $10^{18} \mathrm{eV}$ colliding with microwave-background photons is sufficient to generate electron-positron pairs $p+\gamma_{3 K} \rightarrow p+e^{+}+e^{-}$. As a consequence, the cosmic-ray particles loose energy which leads presumably to a reduction of the flux or a dip in the spectrum between $10^{18}$ and $10^{19} \mathrm{eV}$ [Hill and Schramm(1985), Berezinsky(2005), Berezinsky et al.(2004)].

The effect of both processes on the observed energy spectrum is frequently expressed by a modification factor $f(E)=I_{p}(E) / I_{0}(E)$, describing the ratio 

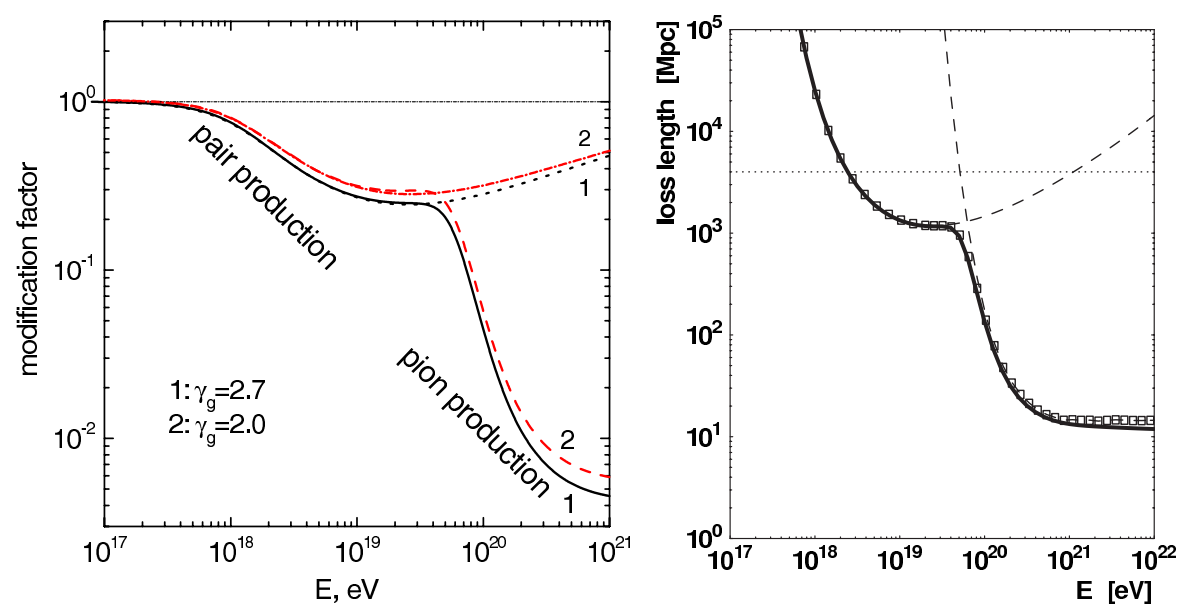

Figure 2: Left: Modification factor $f(E)=I_{p}(E) / I_{0}(E)$ of the cosmic-ray energy spectrum [Aloisio et al.(2007a)]. Right: Loss length for protons for pair production and pion photoproduction [De Marco and Stanev(2005)].

of the observed spectrum $I_{p}$ and the initial spectrum $I_{0}$ as function of energy. The modification factor according to recent calculations is shown in Fig.2 (left) A Aloisio et al.(2007a)]. A twofold structure can be recognized. A depression (the dip) at energies between $10^{18}$ and $10^{19} \mathrm{eV}$ and the GZK feature at energies exceeding $5 \cdot 10^{19} \mathrm{eV}$. The two cases (1 and 2) represent initial spectra with a spectral index of 2.0 and 2.7 , respectively.

The energy loss length for pair production and pion photoproduction is depicted in Fig.2 (right) [De Marco and Stanev(2005)]. Particles with energies above the threshold of the GZK effect can travel less than about $100 \mathrm{Mpc}$ through the Universe, before their energy has decreased to $1 / e$ of their initial value. Or, in other words, particles reaching the Earth at these energies have propagated less than $100 \mathrm{Mpc}$, see also Aharonian and Cronin(1994), and their sources are inside a sphere with this radius

\subsection{Multi Messenger Approach}

It has been discussed that for both scenarios, acceleration and top-down models, hadronic cosmic rays are accompanied by high-energy photons and neutrinos. Also during the propagation of hadronic particles through the Universe high-energy photons and neutrinos are produced. To clarify the origin of the highest-energy particles in the Universe, simultaneous observations are desired of high-energy charged particles, photons, and neutrinos a multi-messenger approach. Thus, the observation of high-energy charged particles, or charged particle astronomy, is complementary to observations in gamma ray astronomy $[\mathrm{Ong}(1998), \widehat{\mathrm{Ong}(2005)]}$ and neutrino astronomy [Spiering(2003), Halzen(2005), Berezinsky(2006), Lipari(2006)]. 
Attention has to be paid on the 'simultaneous' observation: if a charged particle is deflected by an angle $\Theta$ in a (for this estimate simply homogeneous) magnetic field, its path $L_{c h}$ is somewhat longer as compared to the path of a massless neutral particle $L_{\gamma}$. Their relative difference can be approximated as

$$
\mathcal{R}=\frac{L_{c h}}{L_{\gamma}}=\frac{2 \pi \Theta}{360^{\circ} \sin (\Theta)} .
$$

If a charged particle from a source at a distance $L_{\gamma}=100 \mathrm{Mpc}$ is deflected by $\Theta=3^{\circ}$, a value $\mathcal{R}=1+4.6 \cdot 10^{-4}$ is obtained. This corresponds to a difference in the arrival time of a charged particle relative to a photon (both traveling at the speed of light) of about $\Delta T=150 \cdot 10^{3}$ a. Thus, for a simultaneous detection the acceleration processes have to be stable over such a period in time.

\section{Detection Method}

The extremely steeply falling cosmic-ray energy spectrum $\left(\propto E^{-3}\right)$ yields very low fluxes for the highest-energy particles. At the highest energies less than one particle is expected per square kilometer and century. This necessitates huge detection areas and large measuring times. At present, they are only realized in huge ground based installations, registering secondary particles produced in the atmosphere.

\subsection{Extensive air showers}

When high-energy cosmic-ray particles penetrate the Earths atmosphere they interact and generate a cascade of secondary particles, the extensive air showers. Hadronic particles interact and produce new hadronic particles or generate muons and photons through pion decays. Some of the muons may decay into electrons, while the photons and electrons/positrons regenerate themselves in an electromagnetic cascade. The by far dominant particles in a shower are electromagnetic particles (photons, electrons, and positrons). Most of the energy of the primary particle is absorbed in the atmosphere. However, a small fraction of the energy is transported to ground level and may be registered in detectors for electrons, muons, and hadrons. Particles traveling with relativistic speeds through the atmosphere (mostly electrons and positrons) emit Čerenkov light. The shower particles also excite nitrogen molecules in the air which in turn emit fluorescence light. While the Cerenkov light is collimated in the forward direction of the particle, the fluorescence light is emitted isotropically, thus, a shower can be "viewed from aside".

The objective of experiments observing extensive air showers is to determine the properties of the primary particle (energy $E_{0}$, mass $A$, arrival direction). In the energy regime of interest $\left(E>10^{17} \mathrm{eV}\right)$ mainly two methods are applied. Electrons (and positrons) as well as muons reaching ground level are observed in large arrays of detectors and the fluo- 
rescence light is viewed by imaging telescopes. An alternative technique, presently under investigation, is the detection of radio emission from air showers. Electrons and positrons are deflected in the Earths magnetic field and emit synchrotron radiation, which is detected in arrays of dipole antennae [Falcke et al.(2005), van den Berg et al.(2007), Huege and Falcke(2005)].

\subsection{Measuring technique}

The direction of air showers is inferred applying two techniques. The particles in a shower travel with nearly the speed of light through the atmosphere in a thin disc with a thickness of a few meters only. With detectors measuring the arrival time of the particles with a resolution of a few ns the angle of the shower front relative to the ground can be inferred, with the arrival direction being perpendicular to the shower plane. With imaging fluorescence telescopes the shower-detector plane is determined from the observed track in the camera. The orientation of the shower axis in this plane is then obtained by measurements of the arrival time of the photons at the detector. Using two (or more) telescopes to view the same shower allows a three-dimensional reconstruction of the shower axis.

The shower energy is proportional to the number of electrons $N_{e}$ and muons $N_{\mu}$ in the shower. A simple numerical model [Hörandel(2007b)] yields the relations

$$
E_{0}=3.01 \mathrm{GeV} \cdot A^{0.04} \cdot N_{e}^{0.96} \quad \text { and } \quad E_{0}=20 \mathrm{GeV} \cdot A^{-0.11} \cdot N_{\mu}^{1.11}
$$

to estimate the primary energy. This illustrates that measuring $N_{e}$ or $N_{\mu}$ gives a good estimate for the energy almost independent of the particles mass.

With imaging fluorescence telescopes the amount of fluorescence light can be measured as function of depth in the atmosphere. The total amount of light collected is proportional to the shower energy. Using the number of electrons at shower maximum, the number of photons registered per square meter in a detector at a distance $r$ to the maximum of a shower with energy $E_{0}$ can be estimated as

$$
\nu_{\gamma}=\frac{N_{e} X_{0} N_{\gamma}}{4 \pi r^{2}} \approx 790 \frac{\gamma}{\mathrm{m}^{2}} A^{-0.046}\left(\frac{E_{0}}{\mathrm{EeV}}\right)^{1.046} \frac{1}{(r / 10 \mathrm{~km})^{2}},
$$

where $N_{\gamma} \approx 4 \mathrm{\gamma} / \mathrm{m}$ is the fluorescence yield of electrons in air and $X_{0}=$ $36.7 \mathrm{~g} / \mathrm{cm}^{2}$ (or $304 \mathrm{~m}$ at normal pressure) the radiation length. Absorption and scattering in the atmosphere have been neglected in this simple estimate, thus, the equation gives an upper limit for the registered photons.

Experimentally most challenging is the estimation of the mass of the primary particle. Showers induced by light and heavy particles develop differently in the atmosphere. The depth in the atmosphere $X_{\max }$ at which the showers contain a maximum number of particles depends on the primary particles mass

$$
X_{\max }^{A}=X_{\max }^{p}-X_{0} \ln A,
$$


where $X_{\max }^{p}$ is the depth of the shower maximum for proton-induced showers [Matthews(2005), Hörandel(2007b). Experiments measuring the longitudinal shower profile by observations of fluorescence light estimate the mass by measurements of $X_{\max }$.

If a shower develops higher in the atmosphere more particles (mostly electrons) are absorbed on the way to the ground. On the other hand, at high altitudes (with low air densities) charged pions are more likely to decay, thus, yielding more muons. Hence, the electron-to-muon ratio observed at ground level depends on the mass of the primary particle. A Heitler model of hadronic showers [Hörandel(2007b)] yields the relation

$$
\frac{N_{e}}{N_{\mu}} \approx 35.1\left(\frac{E_{0}}{A \cdot 1 \mathrm{PeV}}\right)^{0.15}
$$

This implies that the registered electron-to-muon ratio depends on the energy per nucleon of the primary particle.

\subsection{Cosmic-Ray Detectors}

In the following we describe the most important recent detectors for ultra high-energy cosmic rays.

The AGASA experiment The Akeno Giant Air Shower Array (AGASA) was a scintillator array located in Japan $\left(35^{\circ} \mathrm{N}, 138^{\circ} \mathrm{E}\right)$, covering an area of $100 \mathrm{~km}^{2}$ |Takeda et al.(2003)]. It consisted of 111 scintillation counters to register the electromagnetic shower component. Each station covered $2.2 \mathrm{~m}^{2}$ in area. The scintillator blocks with a thickness of $5 \mathrm{~cm}$ were viewed by a $125 \mathrm{~mm}$ diameter photomultiplier tube. To register the muonic shower component, proportional counters were used with a cross section of $10 \times 10 \mathrm{~cm}^{2}$ and a length of $2 \mathrm{~m}$ or $5 \mathrm{~m}$. The absorber consisted either of a $1 \mathrm{~m}$ thick concrete block, a $30 \mathrm{~cm}$ thick iron plate, or a $5 \mathrm{~cm}$ lead plate above a $20 \mathrm{~cm}$ thick iron plate. The threshold energy for muons is about $0.5 \mathrm{GeV}$. In total, 27 detector stations were installed with areas varying from $2.8 \mathrm{~m}^{2}$ to $20 \mathrm{~m}^{2}$.

The HiRes experiment The High Resolution Fly's Eye experiment (HiRes) was located in Utah, USA $\left(40^{\circ} \mathrm{N}, 112^{\circ} \mathrm{W}\right)$ Abu-Zayyad et al.(2000a). It was the successor of the Fly's Eye experiment [Baltrusaitis et al.(1988)], which pioneered the detection of fluorescence light from air showers. HiRes consisted of two detector sites (Hires I \& II) separated by $12.6 \mathrm{~km}$, providing almost $360^{\circ}$ azimuthal coverage, each. Both telescopes were formed by an array of detector units. The mirrors consisted of four segments and formed a $5.1 \mathrm{~m}^{2}$ spherical mirror. At its focal plane an array of $16 \times 16$ photomultiplier tubes was situated, viewing a solid angle of $16^{\circ} \times 16^{\circ}$. HiRes I consisted of 22 detectors, arranged in a single ring, overlooking between $3^{\circ}$ and $17^{\circ}$ in elevation. This detector used an integrating ADC read-out system, which recorded the photomultiplier tubes' pulse height and time information. 

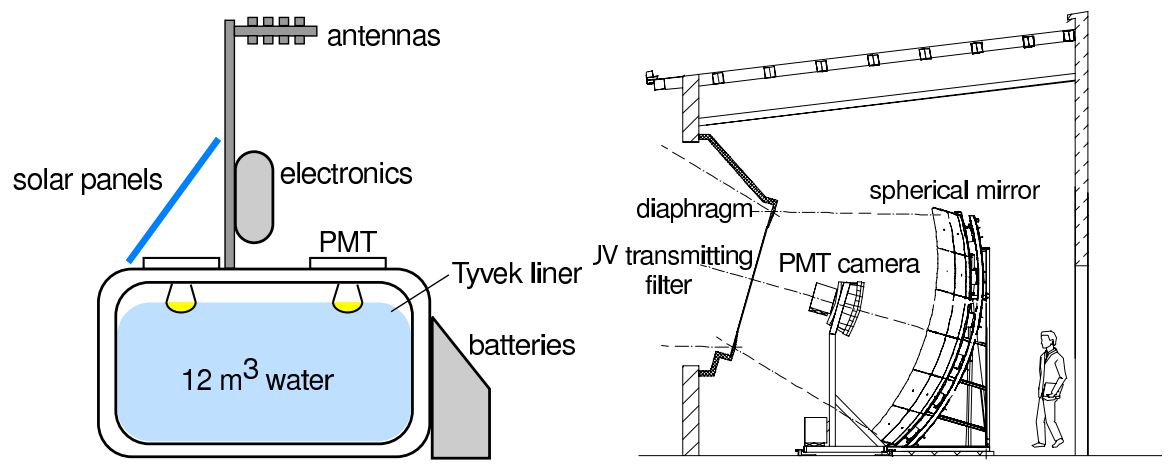

Figure 3: Schematic view of a water Čerenkov detector (left) and a fluorescence telescope (right) of the Pierre Auger Observatory Abraham et al.(2004).

HiRes II comprises 42 detectors, set up in two rings, looking between $3^{\circ}$ and $31^{\circ}$ in elevation. It was equipped with a $10 \mathrm{MHz}$ flash $\mathrm{ADC}$ system, recording pulse height and timing information from its phototubes.

The Pierre Auger Observatory The observatory combines the observation of fluorescence light with imaging telescopes and the measurement of particles reaching ground level in a "hybrid approach" Abraham et al.(2004)]. The southern site (near Malargue, Argentina, $35.2^{\circ} \mathrm{S}, 69.5^{\circ} \mathrm{W}, 1400 \mathrm{~m}$ above sea level) of the worlds largest air shower detector is almost completed. It will consist of 1600 polyethylene tanks set up in an area covering $3000 \mathrm{~km}^{2}$. Each water Čerenkov detector has $3.6 \mathrm{~m}$ diameter and is $1.55 \mathrm{~m}$ high, enclosing a Tyvak liner filled with $12 \mathrm{~m}^{3}$ of high purity water, see Fig.3. The water is viewed by three PMTs (9 in diameter). Signals from the PMTs are read by the electronics mounted locally at each station. Power is provided by batteries, connected to solar panels, and time synchronization relies on a GPS receiver. A radio system is used to provide communication between each station and a central data acquisition system.

Four telescope systems overlook the surface array. A single telescope system comprises six telescopes, overlooking separate volumes of air. A schematic cross-sectional view of one telescope is shown in Fig.3. Light enters the bay through an UV transmitting filter. A circular diaphragm (2.2 m diameter), positioned at the center of curvature of a spherical mirror, defines the aperture of the Schmidt optical system. A $3.5 \mathrm{~m} \times 3.5 \mathrm{~m}$ spherical mirror focuses the light onto a camera with an array of $22 \times 20$ hexagonal pixels. Each pixel has a photomultiplier tube, complemented by light collectors. Each camera pixel has a field of view of approximately $1.5^{\circ}$, a camera overlooks a total field of 


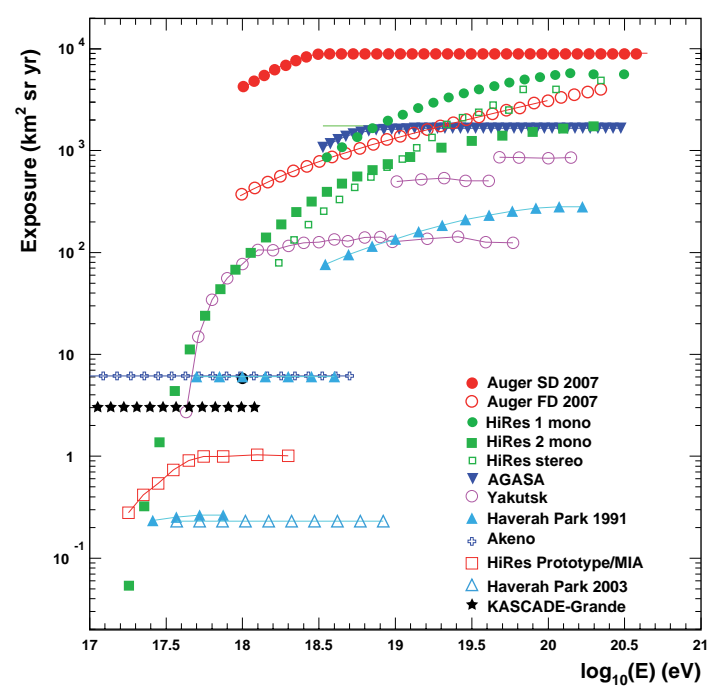

view of $30^{\circ}$ azimuth $\times 28.6^{\circ}$ elevation
Figure 4: Accumulated exposures of various experiments Kampert(2008).

Telescope Array Like the Pierre Auger Observatory, the Telescope array is a hybrid detector, presently under construction in Mullard County, Utah, USA [Fukushima et al.(2007)]. It covers an area of $860 \mathrm{~km}^{2}$ and comprises 576 scintillator stations and three fluorescence detector sites on a triangle with about $35 \mathrm{~km}$ separation, each equipped with twelve fluorescence telescopes.

Accumulated exposures (i.e. experiment aperture times measuring time) for various high-energy experiments are presented in Fig.4 [Kampert(2008)]. For surface arrays the aperture is a function of the detector area and constant with energy. On the other hand, the aperture of fluorescence detectors depends on the shower energy, low energy showers can be seen up to a restricted distance only. This may be illustrated using the approximation (7): the fiducial volume to register $\nu_{\gamma}^{\text {min }}$ photons can be estimated as

$$
V_{f i d} \propto\left(\nu_{\gamma}^{\min }\right)^{-1.5} A^{-0.069} E_{0}^{1.569} .
$$

This shows that the fiducial volume is a function of the primary energy. In this simple approximation there is a small dependence on the mass of the primary particle $(\approx 25 \%$ difference between proton and iron induced showers $)$ and an increase of almost a factor of 40 in the fiducial volume per decade in primary energy. A similar energy dependence can be recognized in Fig. (4) for the various fluorescence detectors. For fluorescence telescopes with a limited field of view in elevation an additional effect occurs: low energy showers penetrate less deep into the atmosphere and thus may have their maximum above the field of view of the telescopes, thus, reducing further the effective aperture. 


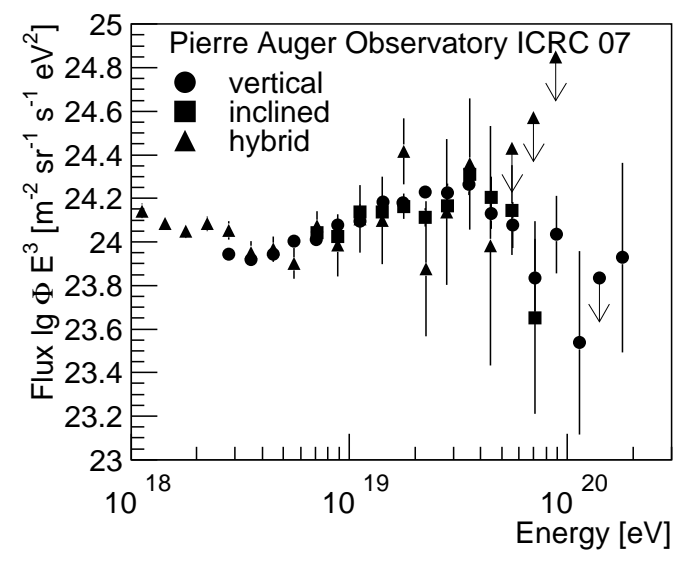

Figure 5: All-particle energy spectra measured by the Pierre Auger Observatory using different reconstruction methods Yamamoto et al.(2007)].

The Pierre Auger Observatory, still under construction, is already the largest cosmic ray detector, the accumulated data exceed the data of all previous experiments. In particular, those of the largest scintillator array (AGASA) and the largest pure fluorescence detector (HiRes). Thus, the Pierre Auger Observatory is expected to measure the properties of ultra high-energy cosmic rays with unprecedented accuracy.

\section{$4 \quad$ Energy Spectrum}

Measurements of the energy spectrum provide important information about the origin of cosmic rays. Over a wide range in energy the all-particle differential energy spectrum is usually described by a power law $d N / d E \propto E^{-\gamma}$. For energies below $10^{15} \mathrm{eV}$ a value for the spectral index $\gamma=-2.7$ has been established by many experiments. The most prominent feature in the all-particle spectrum is the so called knee at an energy of about $4 \cdot 10^{15} \mathrm{eV}$. At this energy the spectral index changes to $\gamma \approx-3.1$. The knee in the all-particle spectrum is caused by the subsequent cut-offs (or knees) of the spectra of individual elements, starting with protons at $E_{k}^{p} \approx 4.5 \cdot 10^{15} \mathrm{eV}$. However, this feature is below the focus of the current article, thus, the reader may be referred to e.g. [Hörandel(2003a), Hörandel(2004), Hörandel(2008)] for a more detailed discussion about galactic cosmic rays and the knee. In the following we focus on energies above $10^{17} \mathrm{eV}$.

Recent energy spectra as obtained by the Pierre Auger Observatory are depicted in Fig. tiplied by $E^{3}$. Different methods are applied to reconstruct the spectra. The first method uses the data from the $3000 \mathrm{~km}^{2}$ surface array. The detection efficiency reaches $100 \%$ for showers with zenith angles less than $60^{\circ}$ for energies above $10^{18.5} \mathrm{eV}$ and for inclined showers $\left(\Theta>60^{\circ}\right)$ above $10^{18.8} \mathrm{eV}$. The signal at $1000 \mathrm{~m}$ from the shower axis is used to estimate the shower energy. To avoid a dependence on interaction models used in air shower simulation 
Table 1: Energy shifts applied to individual experiments as shown in Fig.6.

\begin{tabular}{|c|c|c|}
\hline Experiment & Reference & Energy shift \\
\hline AGASA & |Takeda et al.(2003) & $-22 \%$ \\
\hline Akeno $1 \mathrm{~km}^{2}$ & |Nagano et al.(1984a) & $-4 \%$ \\
\hline Akeno $20 \mathrm{~km}^{2}$ & Nagano et al.(1984b) & $-22 \%$ \\
\hline Auger & [Yamamoto et al.(2007)] & $+20 \%$ \\
\hline Fly's Eye & |Bird et al.(1994) & $-3 \%$ \\
\hline Haverah Park & [Ave et al.(2003)] & $-2 \%$ \\
\hline HiRes-I & [Abbasi et al.(2004a)] & $0 \%$ \\
\hline HiRes-II & |Abbasi et al.(2005)| & $0 \%$ \\
\hline HiRes-MIA & |Abu-Zayyad et al.(2000b) & $+5 \%$ \\
\hline KASCADE-Grande & [Haungs et al.(2008)] & $-7 \%$ \\
\hline MSU & |Fomin et al.(1991)| & $-5 \%$ \\
\hline SUGAR & Anchordoqui and Goldberg(2004) & $0 \%$ \\
\hline Yakutsk T500 & Glushkov et al.(2003) & $-35 \%$ \\
\hline Yakutsk T1000 & Glushkov et al.(2003) & $-20 \%$ \\
\hline
\end{tabular}

codes, an energy estimator is derived based on measured showers: a subset of showers contains so called hybrid events, seen simultaneously by the surface detector array and at least one fluorescence telescope. The fluorescence telescopes provide a nearly model independent calorimetric energy measurement of the showers in the atmosphere. Only a small correction for 'invisible energy' (high-energy muons and neutrinos carrying away energy) has to be applied. This factor amounts to about $10 \%$ and contributes with about $4 \%$ to the systematic error for the energy. The energy calibration thus obtained is applied to all events recorded with the surface detector array. Also inclined events with zenith angles exceeding $60^{\circ}$ have been analyzed, yielding the second spectrum displayed. Finally, a set of showers which have been recorded by at least one surface detector tank and one fluorescence telescope have been analyzed. The resulting energy spectrum reaches energies as low as $10^{18} \mathrm{eV}$, as can be inferred from Fig. 馬, It is interesting to point out that the different spectra have been analyzed independently and agree quite good with each other.

The all-particle energy spectra as obtained by various experiments are compiled in Fig.6. The flux has been multiplied by $E^{3}$. The upper panel shows the original data. The different experiments yield absolute values which differ by almost an order of magnitude in this representation. Nevertheless, the overall shape of the energy spectrum seems to be reflected in all data, irrespective of the absolute normalization. This becomes more obvious when the energy scales are slightly readjusted. Typical systematic uncertainties for the energy scale are of order of $10 \%$ to $30 \%$ in the region of interest. When energy shifts are applied, the results have to be treated with care since the apertures of some experiments change as function of energy (see Fig.4) and this effect has not been taken into account in the procedure used here. 

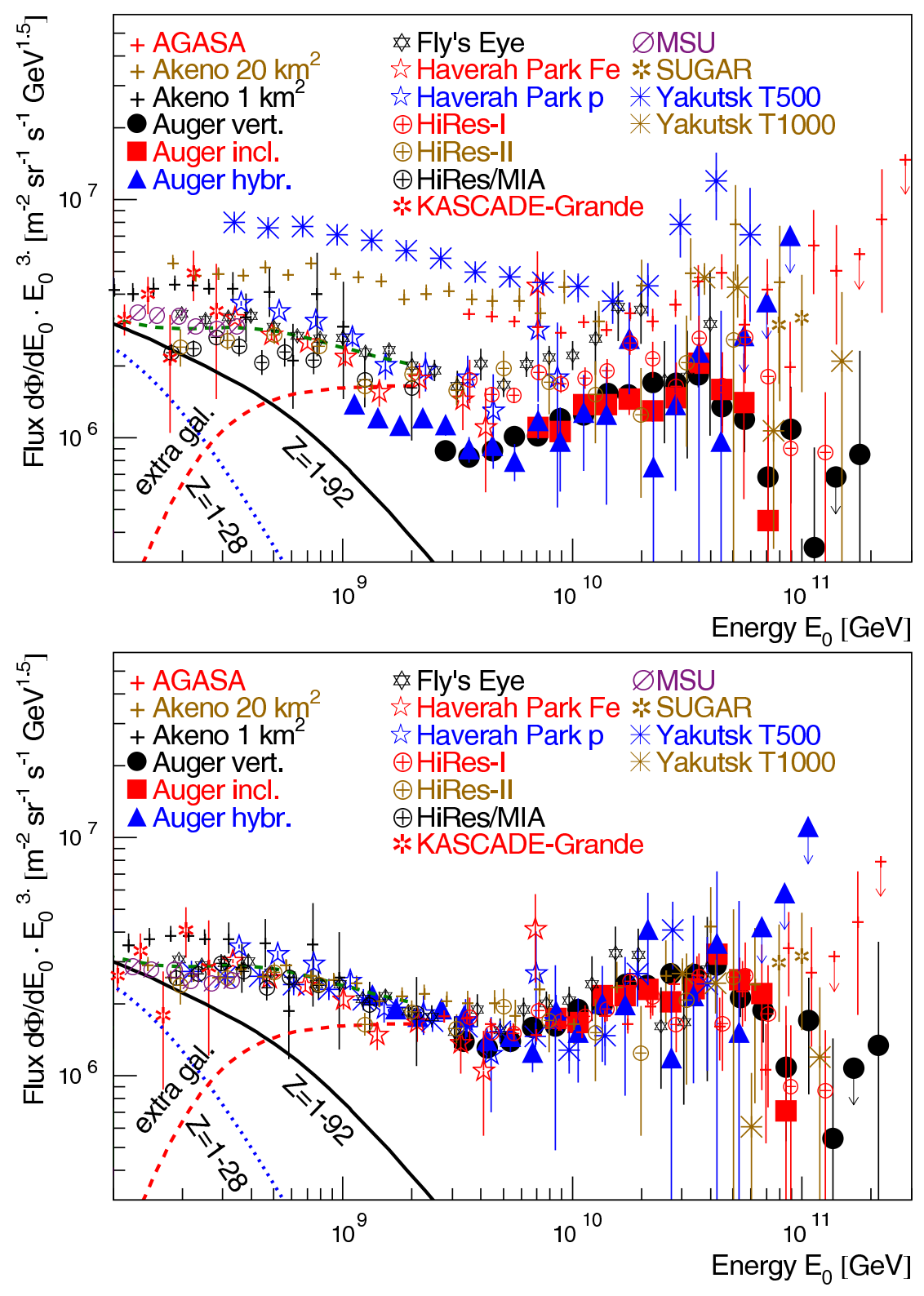

Figure 6: All-particle energy spectra as obtained by different experiments. The top panel shows the original values, in the bottom panel the energy scales of the individual experiments have been adjusted. For references and energy shifts, see Table 1. The lines indicate the end of the galactic component according to the poly-gonato model [Hörandel(2003a)] and a possible contribution of extragalactic cosmic rays. 
In the lower panel of Fig.6 the energy scales of the different experiments have been adjusted to fit the flux according to the poly-gonato model at $10^{18} \mathrm{eV}$. The latter has been obtained through a careful procedure extrapolating the measured spectra for individual elements at low energies [Hörandel(2003a)]. Thus, the normalization applied provides a consistent description from direct measurements ( $10 \mathrm{GeV}$ region) up to the highest energies. The corresponding energy shifts are listed in Table 1.

The normalized spectra agree very well and seem to exhibit a clear shape of the all-particle energy spectrum. Some structures seem to be present in the spectrum. The second knee at about $4 \cdot 10^{17} \mathrm{eV}$, where the spectrum steepens to $\gamma \approx-3.3$ and the ankle at about $4 \cdot 10^{18} \mathrm{eV}$, above this energy the spectrum flattens again to $\gamma \approx-2.7$. Finally, above $4 \cdot 10^{19} \mathrm{eV}$ the spectrum exhibits again a steepening with a spectral index $\gamma \approx-4$ to -5 . The new Auger results help to clarify the situation in this energy region. While the AGASA experiment has reported events beyond the GZK threshold [Takeda et al.(1998)|, the HiRes experiment has reported a detection of the GZK cut-off [Abbasi et al.(2007)]. With the new results, a steeper falling spectrum above $4 \cdot 10^{19} \mathrm{eV}$ is now confirmed.

The second knee possibly marks the end of the galactic component [Hörandel(2003a)]. If the energy spectra for individual elements exhibit knees at energies proportional to their nuclear charge, the heaviest elements in galactic cosmic rays should fall off at an energy of about $92 \cdot E_{k}^{p} \approx 4 \cdot 10^{17} \mathrm{eV}$. An interesting coincidence with the position of the second knee. Different scenarios for the transition from galactic to extragalactic cosmic rays are discussed e.g. in Kampert(2007), Hörandel(2008)]. In the energy region around the ankle a depression is seen in the all-particle flux, also referred to as the dip. It is proposed that this dip is caused by interactions of ultra high-energy particles with the cosmic microwave background, resulting in electron-positron pair production, see Sect.2.2. The steepening in the flux above $4 \cdot 10^{19} \mathrm{eV}$ could be an indication of the GZK effect, i.e. photo-pion production of ultra high-energy cosmic rays with the microwave background, see Sect.2.2, However, for a definite answer also other properties of cosmic rays have to be investigated.

\section{Mass Composition}

The elemental composition of galactic cosmic rays has been discussed elsewhere, e.g. [Hörandel(2003b), Hörandel(2008)]. Above $10^{17} \mathrm{eV}$ the situation is experimentally very challenging, since we are far away in parameter space from collider experiments, where the properties of high-energy interactions are studied in detail. Thus, the air shower models used to interpret the data have to extrapolate over a wide range in parameter space.

The fraction of iron nuclei in cosmic rays as deduced by many experiments has been investigated [Dova et al.(2005)]. No clear conclusion can be drawn about the composition at highest energies. Tension in the interpretation of 
the measured data has been observed as well by the HiRes-MIA experiment Abu-Zayyad et al.(2000c). The observed $X_{\max }$ values exhibit a trend towards a lighter composition as function of energy in the range between $10^{17}$ and $10^{18} \mathrm{eV}$. On the other hand, measured muon densities indicate a very heavy composition in the same energy range.

Methods relying on the measured muon densities, the lateral distribution of Čerenkov light registered at ground level, or geometrically-based methods are rather indirect and depend on certain assumptions and/or interaction models. The most bias free mass estimator is probably a measurement of the depth of the shower maximum $X_{\max }$, preferably with an imaging telescope such as fluorescence detectors. The best way to infer the mass is to measure $X_{\max }$ distributions, rather than average values only. However, unfortunately, also the interpretation of the measured values depends on hadronic interaction models used in air shower simulations.

The average depth of the shower maximum registered by several experiments is plotted in Fig. 7 as function of energy. In the top panel the data are compared to predictions of air shower simulations for primary protons and iron nuclei, using different hadronic interaction models, namely QGSJET 01 [Kalmykov et al.(1997)], QGSJET II-3 [Ostapchenko(2005)], SYBILL 2.1 [Engel et al.(1999)], and DPMJET 2.55 [Ranft(1995)]. The models yield differences in $X_{\max }$ of order of $30 \mathrm{~g} / \mathrm{cm}^{2}$ for iron nuclei and $\approx 50 \mathrm{~g} / \mathrm{cm}^{2}$ for proton induced showers. An overall trend seems to be visible in the data, the measured values seem to increase faster with energy as compared to the model predictions. This implies that the composition becomes lighter as function of energy. Through interactions with the cosmic microwave background heavy nuclei are expected to break up during their propagation through the Universe (GZK effect) and a light composition is expected at the highest energies. However, e.g. the Auger data at the highest energies correspond to a mixed composition for all models displayed.

In the bottom panel of Fig. 7 the measured values are compared to predictions of astrophysical models of the origin of high-energy cosmic rays.

The propagation of high-energy cosmic rays in extragalactic turbulent magnetic fields is considered in Globus et al.(2007)]. The average $X_{\max }$ values are shown for a case, assuming a mixed source composition with an injection spectrum $\propto E^{-2.4}$, a continuous distribution of the sources, and no extragalactic magnetic field. Other cases studied deliver similar results in $X_{\max }$, for details see Globus et al.(2007)].

Different scenarios for the transition from galactic to extragalactic cosmic rays are discussed in [Aloisio et al.(2007b)]. Two scenarios are distinguished, a 'dip' model in which the galactic and extragalactic fluxes equal at an energy below $10^{18} \mathrm{eV}$ and an 'ankle' approach in which both components have equal fluxes at an energy exceeding $10^{19} \mathrm{eV}$. It is proposed that the dip is a consequence of electron-positron pair production, see Sect.2.2. For the 'dip' model a source spectrum $\propto E^{-2.7}$ is assumed and a spectrum $\propto E^{-2}$ for the ankle approach. Cosmic rays have been propagated through an extragalactic magnetic field of $1 \mathrm{nG}$. The resulting average $X_{\max }$ values, based on simula- 

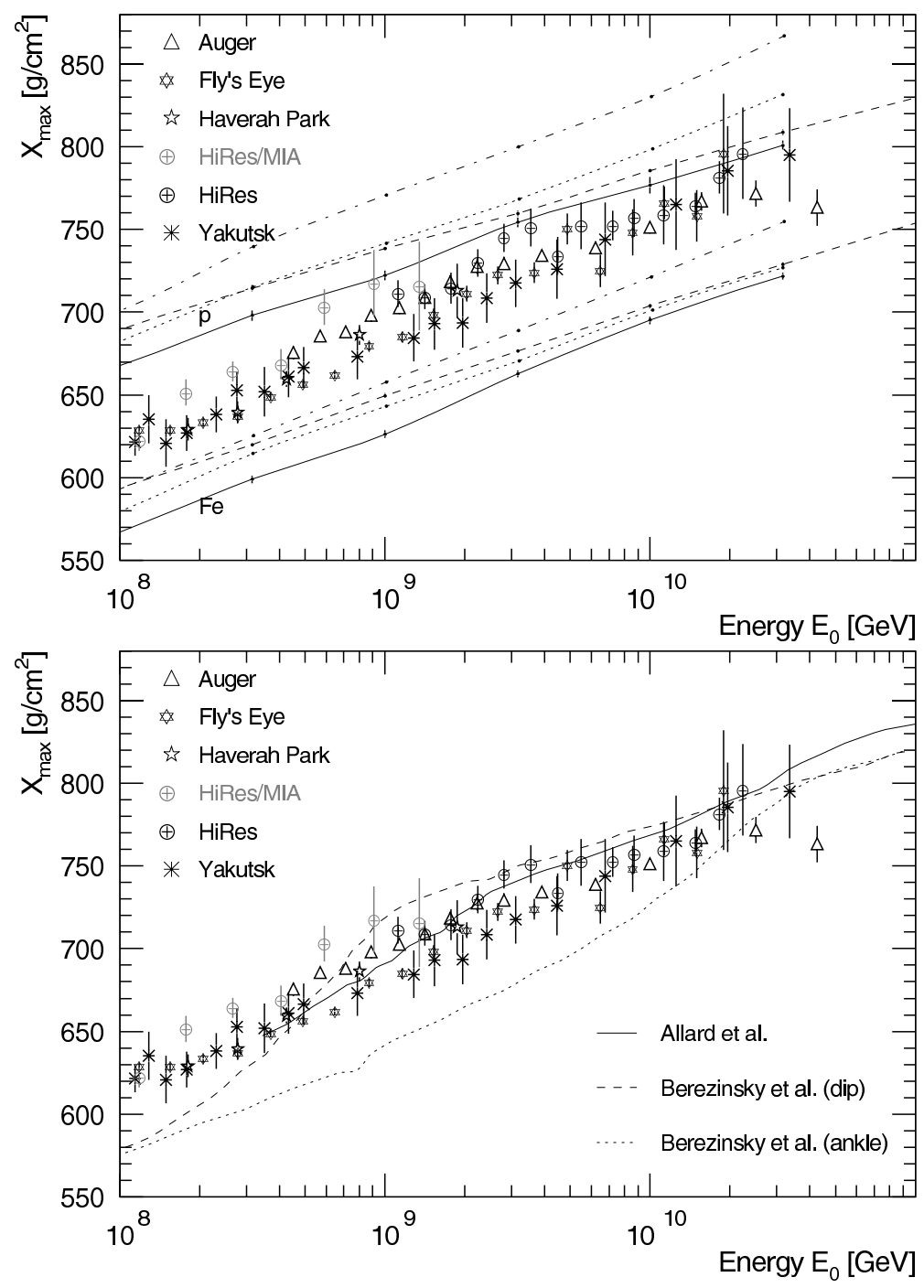

Figure 7: Average depth of the shower maximum $X_{\max }$ as function of energy as measured by the Pierre Auger Observatory [Unger et al.(2007)], as well as the Fly's Eye [Bird et al.(1994)], Haverah Park [Watson(2000)], HiRes/MIA [Abu-Zayyad et al.(2000c)], HiRes [Abu-Zayyad et al.(2000b)], and Yakutsk Knurenko et al.(2001) experiments. Top: measured values are compared to predictions for primary protons and iron nuclei for different hadronic interaction models QGSJET 01 [Kalmykov et al.(1997)] (-), QGSJET II-3 [Ostapchenko(2005)] (---), SYBILL 2.1 [Engel et al.(1999)] ( $\cdots)$, and DPMJET $2.55[\operatorname{Ranft}(1995)](\cdot-\cdot)$. Bottom: comparison to astrophysical models according to [Globus et al.(2007)] (-) as well as [Aloisio et al.(2007b)], for the latter a dip (--) and an ankle $(\cdots)$ scenario are distinguished. 


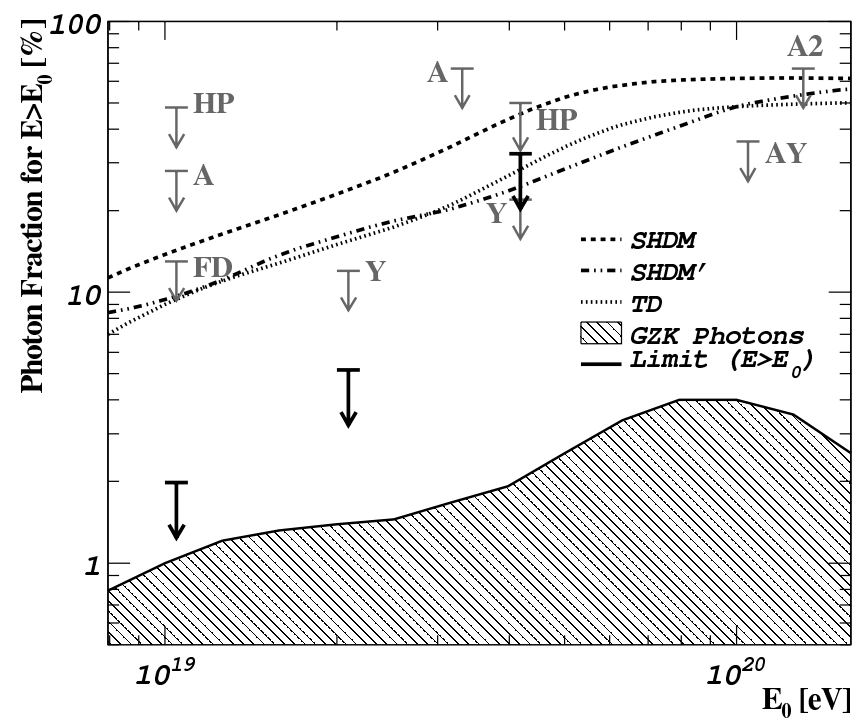

Figure 8: Upper limits on the fraction of photons in the integral cosmicray flux compared to predictions for GZK photons and top-down scenarios Abraham et al.(2007a). Experimental data are from the Auger surface detectors (arrows) [Abraham et al.(2007a)] and a hybrid analysis (FD) |Abraham et al.(2007b)], Haverah Park (HP) [Ave et al.(2002)], AGASA (A) [Shinozaki et al.(2002), Risse et al.(2005)], AGASA and Yakutsk (AY) [Rubtsov et al.(2006)], as well as Yakutsk (Y) [Glushkov et al.(2007)].

tions using the interaction code QGSJET 01 are displayed in the figure.

The figure illustrates that we are entering an era where it should be possible to distinguish between different astrophysical scenarios.

Of great interest is also whether other species than atomic nuclei contribute to the ultra high-energy particle flux.

\subsection{Photon Flux Limit}

Air showers induced by primary photons develop an almost pure electromagnetic cascade. Experimentally they are identified by their relatively low muon content or their relatively deep shower maximum. Since mostly electromagnetic processes are involved in the shower development, the predictions are more reliable and don't suffer from uncertainties in hadronic interaction models. A compilation of recent upper limits on the contribution of photons to the all-particle flux is shown in Fig.8 [Abraham et al.(2007a)]. The best photon limits are the latest results of the Pierre Auger Observatory Abraham et al.(2007a) setting rather strong limits on the photon flux. 


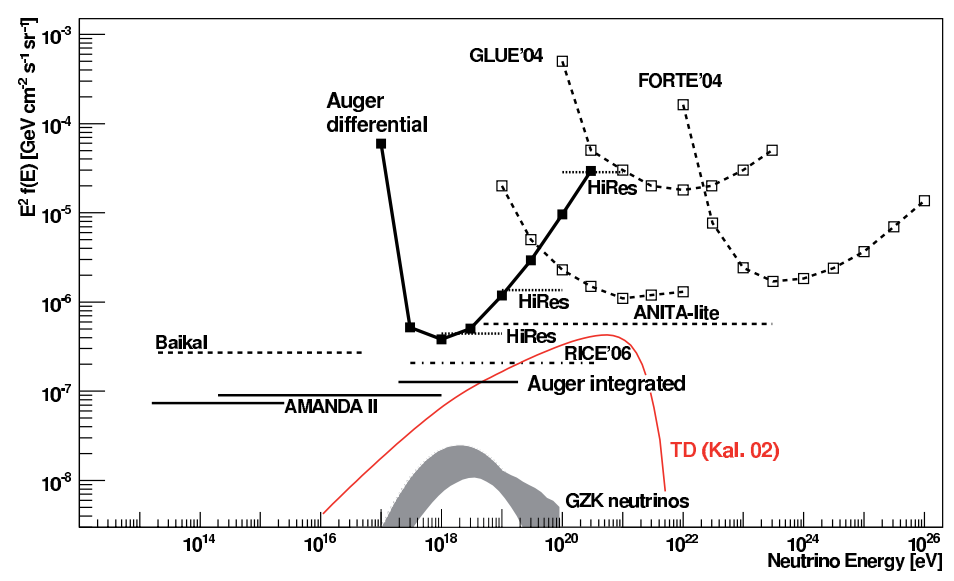

Figure 9: Limits at 90\% confidence level for a diffuse flux of $\nu_{\tau}$ assuming a 1:1:1 ratio of the three neutrino flavors at Earth Abraham et al.(2007c), Kampert(2008)]. The experimental results are compared to predictions for GZK neutrinos and a top-down model [Kalashev et al.(2002)].

They are based on measurements with the Auger surface detectors, taking into account observables sensitive to the longitudinal shower development, the signal rise time, and the curvature of the shower front. The photon fraction is smaller than $2 \%, 5.1 \%$, and $31 \%$ above energies of $10^{19}, 2 \cdot 10^{19}$, and $4 \cdot 10^{19} \mathrm{eV}$, respectively with $95 \%$ confidence level.

In top-down scenarios for high-energy cosmic rays, the particles are decay products of super-heavy particles. This yields relatively high-fluxes of photons predicted by such models. Several predictions are shown in the figure [Aloisio et al.(2004), Ellis et al.(2006)]. These scenarios are strongly disfavored by the recent Auger results.

The upper limits are already relatively close to the fluxes expected for photons originating from the GZK effect [Gelmini et al.(2005)], shown in the figure as shaded area.

\subsection{Neutrino Flux Limit}

The detection of ultra high-energy cosmic neutrinos is a long standing experimental challenge. Many experiments are searching for such neutrinos, and there are several ongoing efforts to construct dedicated experiments to detect them Halzen et al.(2002), Falcke et al.(2004)]. Their discovery would open a new window to the Universe $[\operatorname{Becker}(2007) \mid$. However, so far no ultra high-energy neutrinos have been detected. ${ }^{2}$

\footnotetext{
${ }^{2}$ Neutrinos produced in air showers (atmospheric neutrinos) [Fukuda et al.(1998), in the sun Fukuda et al.(1999), Ahmed et al.(2004)], and during super nova 1987A Hirata et al.(1987), Bionta et al.(1987) have been detected, but are at energies much below our focus.
} 
As discussed above (Sect.22) ultra high-energy cosmic rays are expected to be accompanied by ultra high-energy neutrinos. The neutrinos are produced with different abundances for the individual flavors, e.g. pion decay leads to a ratio $\nu_{e}: \nu_{\mu}=2: 1$. However, due to neutrino oscillations the ratio expected at Earth is $\nu_{\tau}: \nu_{\mu}: \nu_{e}=1: 1: 1$.

To discriminate against the huge hadronic background in air shower detectors, neutrino candidates are identified as nearly horizontal showers with a significant electromagnetic component. The Pierre Auger Observatory is sensitive to Earth-skimming tau-neutrinos that interact in the Earth's crust. Tau leptons from $\nu_{\tau}$ charged-current interactions can emerge and decay in the atmosphere to produce a nearly horizontal shower with a significant electromagnetic component. Recent results from the Pierre Auger Observatory together with upper limits from other experiments are presented in Fig.9. Assuming an $E_{\nu}^{-2}$ differential energy spectrum Auger derives a limit at $90 \%$ confidence level of $E_{\nu}^{2} \mathrm{~d} N_{\nu_{\tau}} / \mathrm{d} E_{\nu}<1.3 \cdot 10^{-7} \mathrm{GeV} \mathrm{cm}^{-2} \mathrm{~s}^{-1} \mathrm{sr}^{-1}$ in the energy range between $2 \cdot 10^{17}$ and $2 \cdot 10^{19} \mathrm{eV}$.

According to top-down models for ultra high-energy cosmic rays a large flux of ultra high-energy neutrinos is expected. As an example, the predictions of a model [Kalashev et al.(2002)] are shown in the figure as well. This model is disfavored by the recent upper limits. It should also be noted that the current experiments are only about one order of magnitude away from predicted fluxes of GZK neutrinos (cosmogenic neutrinos).

\section{Arrival Direction}

The arrival directions of cosmic rays provide an important observable to investigate the sources of these particles. Since charged particles are deflected in magnetic fields, the cosmic-ray flux observed at Earth is highly isotropic. A significant evidence for an anisotropy in the arrival directions would be the most direct hint towards possible cosmic-ray sources. Unfortunately, only limited experimental information is available about both, galactic [Rand and Kulkarni(1989), Vallée(2004)] and extragalactic [Kronberg(1994), Grasso and Rubinstein(2001)| magnetic fields. Selecting particles at the highest energies limits the field of view to distances less than $100 \mathrm{Mpc}$, see Fig.2 (right). This implies two advantages: the number of source candidates is limited and the particles are only slightly deflected since they propagate a restricted distance only.

\subsection{Galactic Center}

The center of our galaxy is an interesting target for cosmic-ray anisotropy studies. It harbors a massive black hole associated with the radio source Sagittarius $\mathrm{A}^{*}$ and a supernova remnant Sagittarius A East. Both are candidates to be powerful cosmic-ray accelerators. The importance is underlined by recent discoveries: the HESS experiment has reported the observation from 


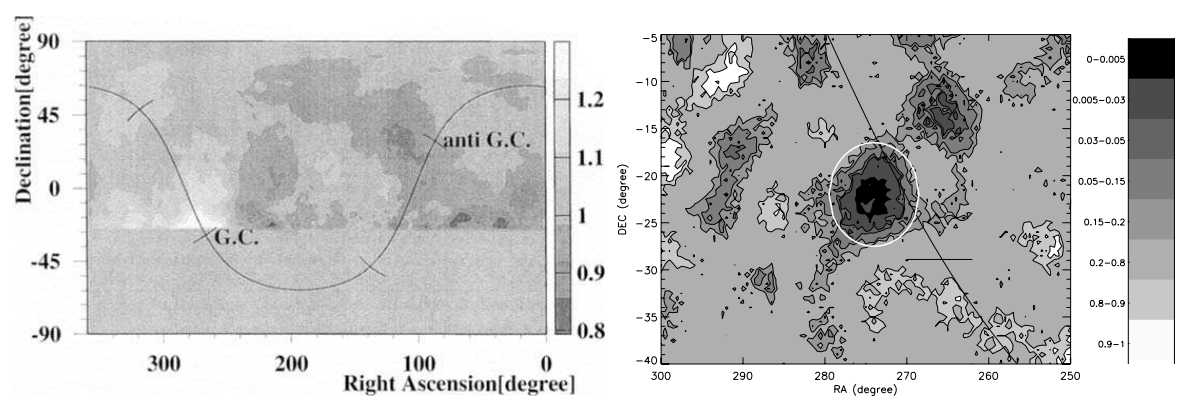

Figure 10: Significance maps of excess/deficit events in equatorial coordinates as measured by the AGASA [Hayashida et al.(1999)] (left) and SUGAR [Bellido et al.(2001)] (right) experiments. The lines in both panels indicate the galactic plane. AGASA: events within a radius of $20^{\circ}$ are summed up in each bin. SUGAR: The white circle with a radius of $5.5^{\circ}$ indicates the error for a point source.

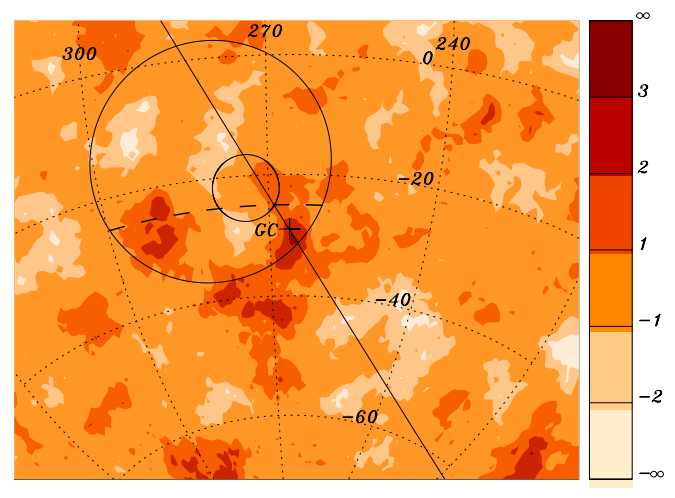

Figure 11: Map of cosmic-ray over-density significances near the Galactic Center as measured by the Pierre Auger Observatory Abraham et al.(2007d). The line indicates the galactic plane and "+" marks the Galactic Center. The circles represent the regions of excess events seen by the AGASA and SUGAR experiments.

$\mathrm{TeV} \gamma$ rays near the location of Sagittarius $\mathrm{A}^{*}$ [Aharonian et al.(2004)] and discovered a region of extended emission from giant molecular clouds in the central 200 pc of the Milky Way [Aharonian et al.(2006)].

Of particular interest to search for anisotropies in cosmic rays is the region around $10^{18} \mathrm{eV}$. At these energies the tail of the galactic component might still contribute significantly to the all-particle spectrum and neutrons from the galactic center can reach the Earth without decaying. Such neutrons would not be deflected by magnetic fields Medina Tanco and Watson(2001), Bossa et al.(2003), Aharonian and Neronov(2005), $\quad$ Crocker et al.(2005), Grasso and Maccione(2005), Biermann et al.(2004)].

The AGASA experiment has investigated anisotropies in the arrival directions of cosmic rays at energies around $10^{18} \mathrm{eV}$, see Fig.10 Hayashida et al.(1999)]. The Galactic Center is just outside the field of view of the experiment. However, an excess in the Galactic-Center region has been 


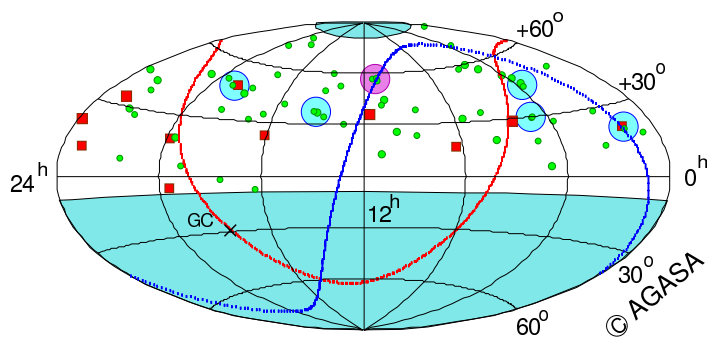

Figure 12: Arrival directions of cosmic rays with energies exceeding $4 \cdot 10^{19} \mathrm{eV}$ in equatorial coordinates as observed by the AGASA experiment. Red squares and green circles represent cosmic rays with energies exceeding $10^{20} \mathrm{eV}$ and $(4-10) \cdot 10^{19} \mathrm{eV}$, respectively. Takeda et al.(1999), AGASA(2003)

detected. Also the SUGAR experiment, located in Australia has reported an excess of events from the region of the Galactic Center at $10^{18} \mathrm{eV}$, see Fig.10 (right) Bellido et al.(2001)]. It should be noted that both findings are on the 3 to $4 \sigma$ level only.

Recently, data from the Pierre Auger Observatory have been searched for anisotropies in the region of the Galactic Center [Abraham et al.(2007d)]. A map of resulting cosmic-ray over-density significances is displayed in Fig.11. The regions were AGASA and SUGAR have found an excess are marked in the figure. With a statistics much greater than those of previous experiments, it has been searched for a point-like source in the direction of Sagittarius A. No significant excess has been found. Also searches on larger angular scales show no abnormally over-dense regions. These findings exclude recently proposed scenarios for a neutron source in the Galactic Center.

\subsection{Clustering of Arrival Directions}

The AGASA experiment has investigated small-scale anisotropies in the arrival directions of cosmic rays [Takeda et al.(1999)]. Above an energy of $4 \cdot 10^{19} \mathrm{eV}$ they have found clusters of events coming from the same direction, see Fig.12, One triplet and three doublets with a separation angle of $2.5^{\circ}$ have been reported, the probability to observe these clusters by a chance coincidence under an isotropic distribution is smaller than $1 \%$.

The HiRes experiment has found no significant clustering at any angular scale up to $5^{\circ}$ for energies exceeding $10 \mathrm{EeV}$ [Abbasi et al.(2004b)]. Combining data from the AGASA, HiRes, SUGAR, and Yakutsk experiments at energies above $40 \mathrm{EeV}$ a hint for a correlation has been found at angular scales around $25^{\circ}$ Kachelriess and Semikoz(2006)].

Also the data of the Pierre Auger Observatory have been searched for clustering in the arrival directions [Mollerarch et al.(2007)]. The autocorrelation function has been analyzed adopting a method, in which a scan over the minimum energy $E$ and the separation angle $\Theta$ is performed 


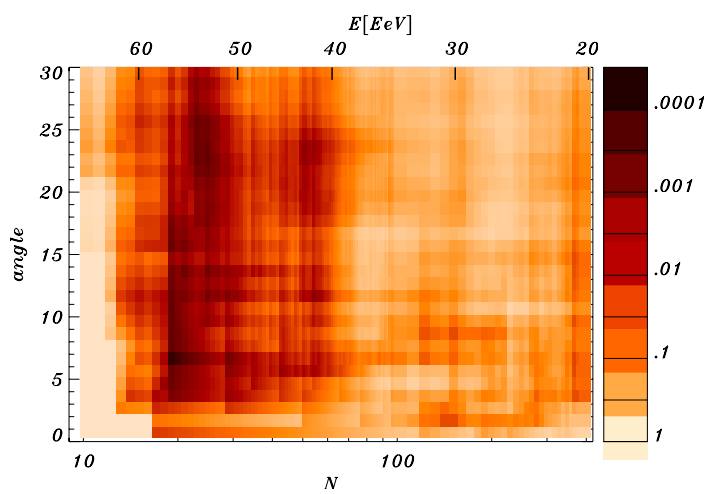

Figure 13: Autocorrelation scan for events with energies above $10^{20} \mathrm{eV}$ recorded with the Pierre Auger Observatory Mollerarch et al.(2007). The chance probability is shown as function of separation angle and threshold energy.

[Finley and Westerhoff(2004)]. For each value of $E$ and $\Theta$ a chance probability is calculated by generating a large number of isotropic Monte Carlo simulations of the same number of events, and computing the fraction of simulations having an equal or larger number of pairs than the data for those parameters. The result is depicted in Fig.13, showing the probability as function of separation angle and threshold energy. A broad region with an excess of correlation appears at intermediate angular scales and large energies. The minimum is found at $7^{\circ}$ for the 19 highest events $(E>57.5 \mathrm{EeV})$, where eight pairs were observed, while one was expected. The fraction of isotropic simulations with larger number of pairs at that angular scale and for that number of events is $P_{\min }=10^{-4}$. The chance probability for this value to arise from an isotropic distribution is $P \approx 2 \cdot 10^{-2}$.

\subsection{Correlation with BL-Lacs}

Interesting candidates as cosmic-ray sources are BL Lacertae objects. They are a sub class of blazars, active galaxies with beamed emission from a relativistic jet which is aligned roughly towards our line of sight. Several experiments have searched for correlations of the arrival directions of cosmic rays with the position of BL Lacs on the sky.

A correlation was found between a subset of BL Lac positions and arrival directions recored by AGASA with energies exceeding $48 \mathrm{EeV}$ and by the Yakutsk experiment at energies above $24 \mathrm{EeV}$ [Tinyakov and Tkachev(2001)]. This correlation and further ones as reported in [Tinyakov and Tkachev(2002), Gorbunov et al.(2002)] between BL Lacs and ultra high-energy cosmic rays registered by the AGASA and Yakutsk experiments were not confirmed by data of the HiRes experiment Abbasi et al.(2006)]. On the other hand, an excess of correlations was found for a subset of BL Lacs and cosmic rays with energies above $10 \mathrm{EeV}$ Abbasi et al.(2006), Gorbunov et al.(2004).

In spring 2007 the number of events recorded by the Pierre Auger Observatory above $10 \mathrm{EeV}$ was six times larger than the data used in previous searches. The correlation hypotheses reported previously have been tested 

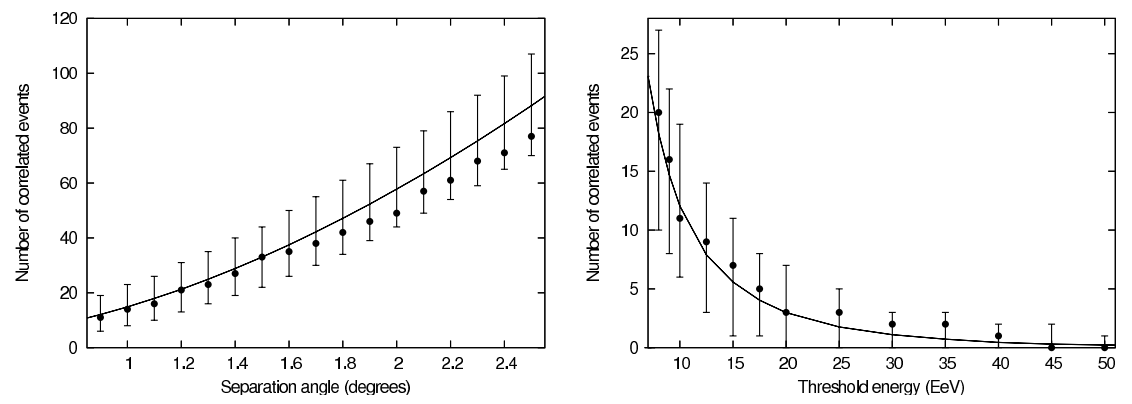

Figure 14: Number of events correlated with confirmed BL Lacs with optical magnitude $m<18$ from the $10^{t h}$ edition of the catalog of quasars and nuclei [Véron-Cetty and Véron(2006)] (points) and average for an isotropic flux (solid line) along with dispersion in $95 \%$ of simulated isotropic sets (bars) [Harari et al.(2007)]. As function of the angular separation (for $E>10 \mathrm{EeV}$, left $)$ and as function of threshold energy $\left(\Theta<0.9^{\circ}\right.$, right $)$.

with the Auger data [Harari et al.(2007)]. Since the southern detector of the Pierre Auger Observatory sees a different part of the sky as compared to the AGASA, HiRes, and Yakutsk experiments, only the 'recipes' could be tested but using different sources on the sky. Non of the previously reported hypotheses could be confirmed, the chance probabilities for the different approaches were found to be slightly smaller than $1 \%$. The correlations search has been extended to a broader range of angular scales and energy thresholds, see Fig.14. It shows the number of correlated events as function of separation angle (left) and energy threshold (right). The curves represent expectations for an isotropic flux. The error bars depict the dispersion within $95 \%$ of simulated isotropic sets. As can be inferred from the figure, the measured data are compatible with an isotropic distribution and they do not confirm earlier findings.

\subsection{Correlation with AGN}

Another interesting set of objects to serve as sources of ultra high-energy cosmic rays are Active Galactic Nuclei (AGN). The radiation from AGN is believed to be a result of accretion on to the super-massive black hole (with $10^{6}$ to $10^{8}$ solar masses) at the center of the host galaxy. AGN are the most luminous persistent sources of electromagnetic radiation in the Universe. An example of an AGN is shown in Fig. 15: Centaurus A is with a distance of $3.4 \mathrm{Mpc}$ one of the closest AGN. The radio lobes are thought to be the result of relativistic jets emerging from the central black hole. Different scenarios related to AGN have been developed, which are supposed to accelerate particles to highest energies, e.g. Biermann and Strittmatter(1987), Rachen and Biermann(1993), Romero et al.(1996), Ostrowski(1999), Lyutikov and Ouyed(1999)].

The arrival directions of cosmic rays as measured by the Pierre 


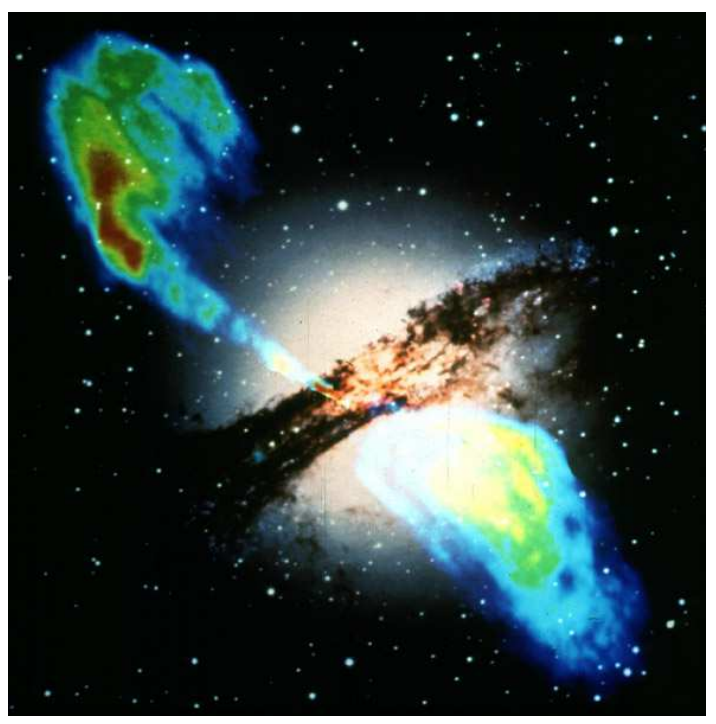

Figure 15: Centaurus A as seen by the Hubble Space Telescope and the VLA radio telescope http://hubblesite.org. The radio lobes extend over a scale of about $10^{\circ}$ along the super-galactic plane.

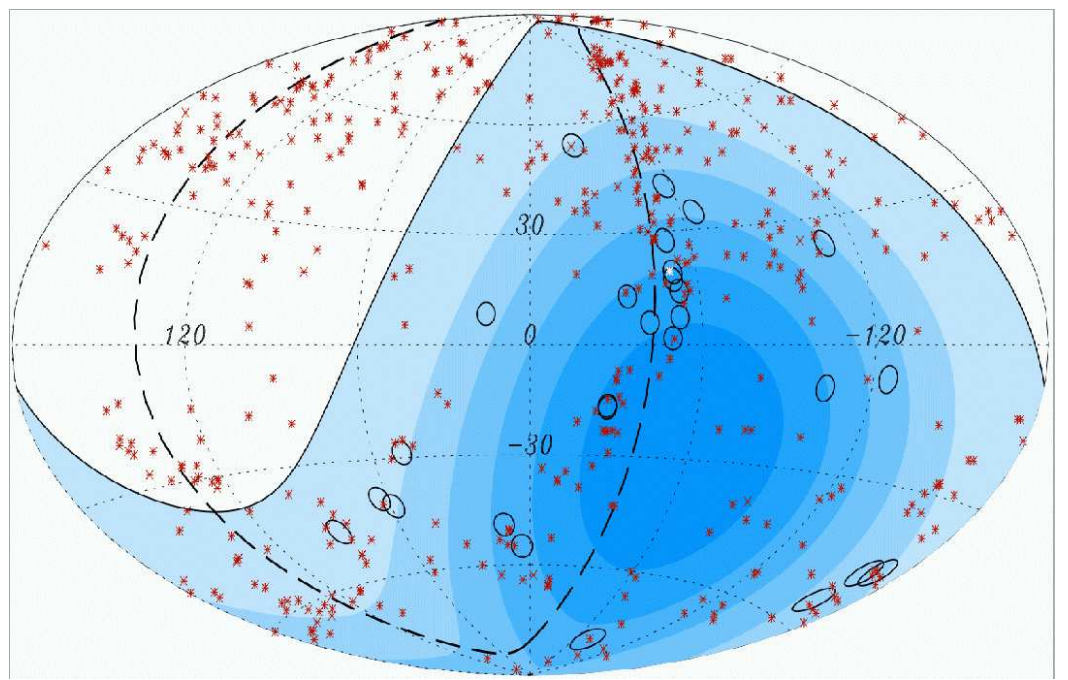

Figure 16: Aitoff projection of the celestial sphere in galactic coordinates with circles of $3.2^{\circ}$ centered at the arrival directions of 27 cosmic rays detected by the Pierre Auger Observatory with energies $E>57 \mathrm{EeV}$ Abraham et al.(2007e)]. The positions of AGN with redshift $z<0.018$ $(D<75 \mathrm{Mpc})$ from the $12^{\text {th }}$ edition of the catalog of quasars and nuclei [Véron-Cetty and Véron(2006) are indicated by the asterisks. The solid line draws the border of the field of view of the southern observatory (for zenith angles $\Theta<60^{\circ}$ ). Darker color indicates larger relative exposure. The dashed line indicates the super-galactic plane. Centaurus A, one of the closest AGN is marked in white. 
Auger Observatory have been correlated with the positions of AGN Abraham et al.(2007e), Abraham et al.(2007f)]. Data taken during the construction of the observatory since January 2004 have been analyzed, corresponding to slightly more than one year of data of the completed observatory. The angular resolution of the detector is better than $1^{\circ}$ at energies above $10^{19} \mathrm{eV}$ [Ave et al.(2007a)]. The positions of AGN according to the $12^{\text {th }}$ edition of the catalog of quasars and nuclei Véron-Cetty and Véron(2006)] within a distance $D$ have been used. A scan has been performed over the distance $D$, a threshold energy $E_{t h}$, and the correlation angle $\Theta$. The best correlation has been found for events with energies exceeding $E_{t h}=57 \mathrm{EeV}$, a maximum distance $D=71 \mathrm{Mpc}$, corresponding to a redshift $z=0.017$, and a correlation angle $\Theta=3.2^{\circ}$. With these parameters 20 out of 27 cosmic rays correlate with at least one of the 442 selected AGN (292 in the field of view of the observatory). Only 5.6 are expected, assuming an isotropic flux. The 27 cosmic rays measured with the highest energies are shown in Fig.16 together with the positions of the AGN. Many of the observed correlated events are aligned with the super-galactic plane. Two events have arrival directions less than $3^{\circ}$ away from Centaurus A. These results indicate clearly that the arrival directions of cosmic rays at highest energies are not isotropic.

A cosmic ray with charge $Z$ e that travels a distance $D$ in a regular magnetic field $B$ is deflected by an angle Abraham et al.(2007f)

$$
\delta \approx 2.7^{\circ} \frac{60 \mathrm{EeV}}{E / Z}\left|\int_{0}^{D}\left(\frac{d x}{\mathrm{kpc}} \times \frac{B}{3 \mu \mathrm{G}}\right)\right| .
$$

Assuming a coherence scale of order $\approx 1 \mathrm{kpc}[\operatorname{Stanev(1997)]}$ for the regular component of the galactic magnetic field, the deflection angle is a few degrees only for protons with energies larger than $60 \mathrm{EeV}$. This illustrates that the observed angular correlations are reasonable, but one has to keep in mind the limited knowledge about galactic magnetic fields. The angular scale of the observed correlations also implies that intergalactic magnetic fields along the line of sight to the sources do not deflect cosmic-ray trajectories by much more than a few degrees. The root mean square deflection of cosmic rays with charge $Z e$, traveling a distance $D$ in a turbulent magnetic field with coherence length $L_{c}$ is Abraham et al.(2007f)

$$
\delta_{r m s} \approx 4^{\circ} \frac{60 \mathrm{EeV}}{E / Z} \frac{B_{r m s}}{\mathrm{nG}} \sqrt{\frac{D}{100 \mathrm{Mpc}}} \sqrt{\frac{L_{c}}{1 \mathrm{Mpc}}} .
$$

As information on intergalactic magnetic fields is very sparse, the correlations observed can be used to constrain models of turbulent intergalactic magnetic fields. Within the observed volume they should be such that in most directions $B_{r m s} \sqrt{L_{c}} \leq 10^{-9} \mathrm{G} \sqrt{\mathrm{Mpc}}$. In the future the Pierre Auger Observatory will collect more data and more than one event per source should be detected. It should then be possible to use the data itself to set constraints on magnetic field models. 
It should be noted that the findings by the Pierre Auger Observatory imply that the sources of the highest-energy-cosmic rays are spatially distributed like AGN. The actual acceleration sites could be the AGN itself or other candidates with the same spatial distribution as AGN.

\section{Discussion and Outlook}

"How do cosmic accelerators work and what are they accelerating?" is one of eleven science questions for the new century in physics and astronomy [National Research Council(2003)]. In the last few years important progress has been made in measuring the properties of ultra high-energy cosmic rays. In particular, the results of the Pierre Auger Observatory have significantly contributed to an improvement in understanding the origin of the highestenergy particles in the Universe. The discovery of correlations between the arrival directions of cosmic rays and the positions of AGN was among the most important scientific breakthroughs in 2007 for several science media organizations, see [www.auger.org/news/top_news_2007.html].

The must important findings discussed in this overview may be summarized as follows. The structures in the energy spectrum at highest energies seem to become more clear. In particular, there seems to be evidence for a steeper falling spectrum above $4 \cdot 10^{19} \mathrm{eV}$ (Figs. 5 and 6). The question arises whether this steepening is due to the GZK effect or due to the maximum energy achieved during the acceleration processes. The most convincing evidence for the existence of the GZK effect is provided by the correlations of the arrival directions with AGN. They occur sharply above an energy of $57 \mathrm{EeV}$. At this energy, the flux measured by the Pierre Auger Observatory is about $50 \%$ lower than expected from a power law extrapolation from lower energies, see Fig. 5 . Thus, there seems to be a connection between the steepening in the spectrum and the AGN correlation.

The correlations occur on an angular scale of about $3.2^{\circ}$. This indicates that the particles are deflected marginally only. In turn, this implies they should be light particles, with low $Z$, see (11) and (12). However, there is some tension between this expectation and the measurements of the average depth of the shower maximum $X_{\max }$ (Fig.7). The data at $4 \cdot 10^{19} \mathrm{eV}$ are compatible with a mixed composition. But, since the correlations occur relatively sharp above $57 \mathrm{EeV}$, some dramatic change in composition above this energy can also not be excluded.

The correlation implies that the sources of ultra high-energy particles are in our cosmological neighborhood $(D<75)$. The GZK horizon, defined as the distance from Earth which contains the sources that produce $90 \%$ of the protons that arrive with energies above a certain threshold is $90 \mathrm{Mpc}$ at $80 \mathrm{EeV}$ and $200 \mathrm{Mpc}$ at $60 \mathrm{EeV}$ [Harari et al.(2006)]. There seems to be a slight mismatch between these numbers and the Auger findings. Shifting upward the Auger energy scale by about $30 \%$, as indicated by some simulations of the reconstruction procedures [Engel et al.(2007)], a better agreement between 
the predicted GZK horizon and energy threshold with the observed data could be achieved [Abraham et al.(2007f)].

The biggest uncertainty in the absolute energy scale of the Pierre Auger Observatory is the knowledge of the fluorescence yield. At present, intensive efforts are conducted by various groups to precisely determine the fluorescence yield of electrons in air [Arqueros et al.(2008)]. Attention is paid to the dependence of the yield on atmospheric parameters, like pressure, temperature, and humidity. In particular, upcoming results from the AIRFLY experiment [Ave et al.(2007b), Privitera et al.(2007)] are expected to significantly reduce the uncertainties of the energy scale for fluorescence detectors.

The correlation between the arrival directions and the positions of AGN sets constraints on models for the acceleration of ultra high-energy particles. The results imply that the spatial distribution of sources is correlated with the distribution of AGN. Thus, already some scenarios are strongly disfavored. Ruled out are models proposing sources in our Galaxy, like neutron stars [Blasi et al.(2000)], pulsars [Bednarek(2003)], and black holes [Dar and Plaga(1999)|. Models for sources in the galactic halo are also ruled out such as top-down scenarios with decaying super-heavy particles Berezinsky et al.(1997), Kuz'min and Rubakov(1998), Birkel and Sarkar(1998)]. These models are also severely constraint by the upper limits on the photon flux (Fig.8) and the neutrino flux (Fig.99). Within the next years measurements of photons and neutrinos produced in the GZK effect seem to be in reach. Their detection would be an important and complementary information about the origin and propagation of ultra high-energy cosmic rays.

In summary, the acceleration of ultra high-energy particles in AGN seems to be very attractive, different scenarios have been proposed, e.g. Biermann and Strittmatter(1987), Rachen and Biermann(1993), Romero et al.(1996), Ostrowski(1999), Lyutikov and Ouyed(1999)|. However, other sources with a similar spatial distribution are not excluded.

With the energy density estimated in Sect.2.1 we obtain a total cosmic ray power of about $9.7 \cdot 10^{43} \mathrm{erg} / \mathrm{s}$ within a sphere $(r=75 \mathrm{Mpc})$ seen by the Pierre Auger Observatory at the highest energies. The typical power in the jets of AGN is of order of $10^{44}$ to $10^{46} \mathrm{erg} / \mathrm{s}$ Körding et al.(2008)]. If we assume about $10 \%$ of this power is converted into cosmic rays, about 1 to 10 sources are needed to sustain the power of the observed extragalactic cosmic ray flux within a distance of $75 \mathrm{Mpc}$ from Earth. If the efficiency is slightly smaller, the number of sources required is correspondingly slightly larger. ${ }^{3}$ If the sources of the highest-energy particles are indeed related to AGN, the number of correlated events seen by the Pierre Auger Observatory seems to be of the right order of magnitude and one expects to see in future more events from the same sources.

When the number of correlated events found in the Auger data is compared to expectations for the AGASA and HiRes experiments, one has to be aware

\footnotetext{
${ }^{3}$ Based on statistical arguments a minimum number of sources $\geq 61$ has been estimated Abraham et al.(2007f).
} 
of the different energy scales, see Table 1. The energy scales of the AGASA and HiRes experiment are shifted relative to the Pierre Auger Observatory by about $42 \%$ and $20 \%$, respectively. If the Auger prescription is applied to the data of these experiments, the energy threshold has to be adjusted correspondingly.

In the next years several experiments focus on the exploration of the energy region of the transition from galactic to extragalactic cosmic rays $\left(10^{17}\right.$ to $10^{18} \mathrm{eV}$ ). The $0.5 \mathrm{~km}^{2}$ KASCADE-Grande experiment [Navarra et al.(2004)] is taking data since 2004 [Chiavassa et al.(2005)]. The Ice Cerenkov detector Ice Cube [Kestel et al.(2004)] at the South Pole and its $1 \mathrm{~km}^{2}$ surface air shower detector Ice Top [Gaisser et al.(2003)] are under construction. In January 200840 Ice Cube strings and 40 surface detectors have been deployed, which implies the set-up is already $50 \%$ completed. Further experiments are the Telescope Array [Fukushima et al.(2007)] and its low energy extension TALE, as well as extensions of the Pierre Auger Observatory to lower energies [Kalges et al.(2007)]. With this new high-quality data more detailed information will be available on the energy spectrum and the composition and it should be possible to distinguish between different scenarios for the transition from galactic to extragalactic cosmic rays.

A promising complementary detection method for high-energy cosmic rays is the measurement of radio emission from air showers. This method provides three-dimensional information about air showers, similar to the fluorescence technique, but with the advantage of a much higher duty cycle. In the next years air showers are expected to be detected with the LOFAR radio observatory $[\overline{\operatorname{LOFAR}(2007)]}$. An extensive research and development program is conducted in the Pierre Auger Collaboration with the goal to build a $20 \mathrm{~km}^{2}$ radio antennae array van den Berg et al.(2007)].

The southern site of the Pierre Auger Observatory covers only a part of the whole sky, see Fig.16. Since the distribution of matter in the Universe is different in the parts seen from the northern and southern hemispheres it is important to observe the whole sky. The Northern Auger Observatory is designed to complete and extend the investigations begun in the South [Nitz et al.(2007)]. To unambiguously identify the sources of the highestenergy cosmic rays requires collecting many more events in spite of the steeply falling energy spectrum. The planned Northern site will be located in Southeast Colorado, USA, having an instrumented area several times the area of Auger South.

The Northern Observatory needs unrestricted support now, it is the next step in exploring the high-energy Universe in the upcoming years. With the completed Pierre Auger Observatory, with its Southern and Northern sites operated simultaneously, an exciting future in astroparticle physics is ahead of us. It will establish charged particle astronomy on the whole sky and will provide high-accuracy data to test astrophysical models of the origin of ultra high-energy cosmic rays. In addition, it will improve our understanding of fundamental physics. The data will give insight into topics like the existence of 
vacuum Čerenkov radiation, the smoothness of space, and tests of Lorentz invariance [Klinkhammer and Risse(2008), Galaverni and Sigl(2007)]. Already now, the existing Auger data set stringent limits on theories.

\section{Acknowledgment}

I would like to thank the organizers of "Cosmic Matter" for the excellent meeting, bringing together the astroparticle physics and astronomy communities and for their invitation to give an overview talk. Many thanks to John Harton for critically reading the manuscript. I'm grateful to my colleagues from the Pierre Auger Observatory as well as the LOFAR, LOPES, KASCADEGrande, and TRACER experiments for fruitful discussions.

\section{References}

[Abbasi et al.(2004a)] Abbasi, R., et al., 2004a. Phys. Rev. Lett. 92, 151101.

[Abbasi et al.(2004b)] Abbasi, R., et al., 2004b. Astrophys. J. 610, L73.

[Abbasi et al.(2005)] Abbasi, R., et al., 2005. Astropart. Phys. 23, 157.

[Abbasi et al.(2006)] Abbasi, R., et al., 2006. Astrophys. J. 636, 680.

[Abbasi et al.(2007)] Abbasi, R., et al., 2007. astro-ph/0703099

[Abraham et al.(2004)] Abraham, J., et al., 2004. Nucl. Instr. \& Meth. A 523, 50.

[Abraham et al.(2007a)] Abraham, J., et al., 2007a. arXiv:0712.1147, submitted to Astropart. Phys.

[Abraham et al.(2007b)] Abraham, J., et al., 2007b. Astropart. Phys. 27, 155.

[Abraham et al.(2007c)] Abraham, J., et al., 2007c. arXiv:0712.1909, submitted to Phys. Rev. Lett.

[Abraham et al.(2007d)] Abraham, J., et al., 2007d. Astropart. Phys. 27.

[Abraham et al.(2007e)] Abraham, J., et al., 2007e. Science 318, 938.

[Abraham et al.(2007f)] Abraham, J., et al., 2007f. arXiv:0712.2843

[Abu-Zayyad et al.(2000a)] Abu-Zayyad, T., et al., 2000a. Nucl. Instr. \& Meth. A $450,253$.

[Abu-Zayyad et al.(2000b)] Abu-Zayyad, T., et al., 2000b. Astrophys. J. 557, 686.

[Abu-Zayyad et al.(2000c)] Abu-Zayyad, T., et al., 2000c. Phys. Rev. Lett. 84, 4276.

[AGASA(2003)] AGASA, 2003. http://www-akeno.icrr.u-tokyo.ac.jp/AGASA/

[Aharonian and Cronin(1994)] Aharonian, F., Cronin, J., 1994. Phys. Rev. D 50, 1892.

[Aharonian and Neronov(2005)] Aharonian, F., Neronov, A., 2005. Astrophys. J. 619, 306.

[Aharonian et al.(2004)] Aharonian, F., et al., 2004. Astron. \& Astroph. 425, L13.

[Aharonian et al.(2006)] Aharonian, F., et al., 2006. Nature 439, 695.

[Ahmed et al.(2004)] Ahmed, S., et al., 2004. Phys. Rev. Lett. 92, 181301. 
[Aloisio et al.(2004)] Aloisio, R., et al., 2004. PRD 69, 094023.

[Aloisio et al.(2007a)] Aloisio, R., et al., 2007a. Astropart. Phys. 27, 76.

[Aloisio et al.(2007b)] Aloisio, R., et al., 2007b. arXiv:0706.2834

[Anchordoqui and Goldberg(2004)] Anchordoqui, L., Goldberg, H., 2004. Phys. Lett. B 583, 213.

[Arqueros et al.(2008)] Arqueros, F., Hörandel, J., Keilhauer, B. (Eds.), 2008. Proceedings of the 5th Fluorescence Workshop, El Escorial, Spain. Nuclear Instr. and Meth., in press.

[Ave et al.(2002)] Ave, M., et al., 2002. Phys. Rev. D 65, 063007.

[Ave et al.(2003)] Ave, M., et al., 2003. Astropart. Phys. 19, 47.

[Ave et al.(2007a)] Ave, M., et al., 2007a. Proc. 30th Int. Cosmic Ray Conf., Merida, in press (arXiv:0709.2125).

[Ave et al.(2007b)] Ave, M., et al., 2007b. Astropart. Phys. 28, 41.

[Baltrusaitis et al.(1988)] Baltrusaitis, R., et al., 1988. Nucl. Instr. \& Meth. A 264, 87.

[Becker(2007)] Becker, J., 2007. arXiv:0710.1557.

[Bednarek(2003)] Bednarek, W., 2003. Mon. Not. R. Astron. Soc. 345, 847.

[Bellido et al.(2001)] Bellido, J., et al., 2001. Astropart. Phys. 15, 167.

[Berezinsky(2005)] Berezinsky, V., 2005. astro-ph/0509069

[Berezinsky(2006)] Berezinsky, V., 2006. Nucl. Phys. B (Proc. Suppl.) 151, 260.

[Berezinsky et al.(1997)] Berezinsky, V., et al., 1997. Phys. Rev. Lett. 79, 4302.

[Berezinsky et al.(2004)] Berezinsky, V., et al., 2004. Astropart. Phys. 21, 617.

[Bergman and Belz(2007)] Bergman, D., Belz, J., 2007. J. Phys. G: Nucl. Part. Phys. 34, R359.

[Bhattacharjee and Sigl(2000)] Bhattacharjee, P., Sigl, G., 2000. Phys. Rep. 327, 109.

[Biermann and Strittmatter(1987)] Biermann, P., Strittmatter, P., 1987. Astrophys. J. 322, 643 .

[Biermann et al.(2004)] Biermann, P., et al., 2004. Astrophys. J. 604, L24.

[Bionta et al.(1987)] Bionta, R., et al., 1987. Phys. Rev. Lett. 54.

[Bird et al.(1994)] Bird, D., et al., 1994. Astrophys. J. 424, 491.

[Birkel and Sarkar(1998)] Birkel, M., Sarkar, S., 1998. Astropart. Phys. 9, 297.

[Blasi et al.(2000)] Blasi, P., et al., 2000. Astrophys. J. 533, L123.

[Bossa et al.(2003)] Bossa, M., et al., 2003. J. Phys. G: Nucl. Part. Phys. 29, 1409.

[Chiavassa et al.(2005)] Chiavassa, A., et al., 2005. Proc. 29th Int. Cosmic Ray Conf., Pune 6, 313.

[Crocker et al.(2005)] Crocker, R., et al., 2005. Astrophys. J. 622, 892.

[Dar and Plaga(1999)] Dar, A., Plaga, R., 1999. Astron. \& Astroph. 349, 259.

[De Marco and Stanev(2005)] De Marco, D., Stanev, T., 2005. Phys. Rev. D 72, 081301. 
[Dova et al.(2005)] Dova, M., et al., 2005. Proc. 29th Int. Cosmic Ray Conf., Pune 7,275 .

[Ellis et al.(2006)] Ellis, J., et al., 2006. PRD 74, 115003.

[Engel et al.(1999)] Engel, R., et al., 1999. Proc. 26th Int. Cosmic Ray Conf., Salt Lake City 1, 415.

[Engel et al.(2007)] Engel, R., et al., 2007. Proc. 30th Int. Cosmic Ray Conf., Merida, in press (arXiv:0706.1921).

[Falcke et al.(2004)] Falcke, H., et al., 2004. New Astronomy Reviews 48, 1487.

[Falcke et al.(2005)] Falcke, H., et al., 2005. Nature 435, 313.

[Fichtner(2005)] Fichtner, H., 2005. Proc. 29th Int. Cosmic Ray Conf., Pune 10, 377.

[Finley and Westerhoff(2004)] Finley, C., Westerhoff, S., 2004. Astropart. Phys., 359.

[Fomin et al.(1991)] Fomin, Y., et al., 1991. Proc. 22nd Int. Cosmic Ray Conf., Dublin 2, 85 .

[Fukuda et al.(1998)] Fukuda, Y., et al., 1998. Phys. Lett. B 436, 33.

[Fukuda et al.(1999)] Fukuda, Y., et al., 1999. Phys. Rev. Lett. 82, 1810.

[Fukushima et al.(2007)] Fukushima, M., et al., 2007. Proc. 30th Int. Cosmic Ray Conf., Merida, in press.

[Gaisser(1997)] Gaisser, T., 1997. astro-ph/9707283

[Gaisser(2006)] Gaisser, T., 2006. astro-ph/0608553

[Gaisser and Stanev(2006)] Gaisser, T., Stanev, T., 2006. Nucl. Phys. A 777, 98.

[Gaisser et al.(2003)] Gaisser, T., et al., 2003. Proc. 28th Int. Cosmic Ray Conf., Tsukuba 2, 1117.

[Galaverni and Sigl(2007)] Galaverni, M., Sigl, G., 2007. arXiv:0708.1737.

[Gelmini et al.(2005)] Gelmini, G., et al., 2005. astro-ph/0506128.

[Ginzburg and Syrovatskii(1964)] Ginzburg, V., Syrovatskii, S., 1964. The origin of cosmic rays. Pergamon Press.

[Globus et al.(2007)] Globus, N., et al., 2007. arXiv:0709.1541

[Glushkov et al.(2003)] Glushkov, A., et al., 2003. Proc. 28th Int. Cosmic Ray Conf., Tsukuba 1, 389.

[Glushkov et al.(2007)] Glushkov, A., et al., 2007 85, 163.

[Gorbunov et al.(2002)] Gorbunov, D., et al., 2002. Astrophys. J. 577, L93.

[Gorbunov et al.(2004)] Gorbunov, D., et al., 2004 80, 145.

[Grasso and Maccione(2005)] Grasso, D., Maccione, L., 2005. Astropart. Phys. 24, 273.

[Grasso and Rubinstein(2001)] Grasso, D., Rubinstein, H., 2001. Phys. Rep. 348, 163.

[Greisen(1966)] Greisen, K., 1966. Phys. Rev. Lett. 16, 748.

[Halzen(2005)] Halzen, F., 2005. astro-ph/0506248. 
[Halzen(2006)] Halzen, F., 2006. astro-ph/0604441.

[Halzen et al.(2002)] Halzen, F., et al., 2002. Reports on Progress in Physics 65, 1025.

[Harari et al.(2006)] Harari, D., et al., 2006. J. Cosmol. \& Astropart. Phys. 11, 012.

[Harari et al.(2007)] Harari, D., et al., 2007. Proc. 30th Int. Cosmic Ray Conf., Merida, in press (arXiv:0706.1715).

[Haungs et al.(2008)] Haungs, A., et al., 2008. Nucl. Phys. B (Proc. Suppl.) 175-176, 354.

[Hayashida et al.(1999)] Hayashida, N., et al., 1999. Astropart. Phys. 10, 303.

[Heber(2005)] Heber, B., 2005. Int. J. Mod. Phys. A 20, 6621.

[Hess(1912)] Hess, V., 1912. Phys. Zeitschr. 13, 1084.

[Hill and Schramm(1983)] Hill, C., Schramm, D., 1983. Phys. Lett. B 131, 247.

[Hill and Schramm(1985)] Hill, C., Schramm, D., 1985. Phys. Rev. D 31, 564.

[Hillas(1984)] Hillas, A., 1984. Ann. Rev. of Astron. and Astroph. 22, 425.

[Hirata et al.(1987)] Hirata, K., et al., 1987. Phys. Rev. D 58, 1490.

[Hörandel(2003a)] Hörandel, J., 2003a. Astropart. Phys. 19, 193.

[Hörandel(2003b)] Hörandel, J., 2003b. J. Phys. G: Nucl. Part. Phys. 29, 2439.

[Hörandel(2004)] Hörandel, J., 2004. Astropart. Phys. 21, 241.

[Hörandel(2007a)] Hörandel, J., 2007a. arXiv:0710.4909 (Nuclear Instruments and Methods A in press).

[Hörandel(2007b)] Hörandel, J., 2007b. Mod. Phys. Lett. A 22, 1533.

[Hörandel(2008)] Hörandel, J., 2008. Adv. Space Res. 41, 442.

[Huege and Falcke(2005)] Huege, T., Falcke, H., 2005. Astropart. Phys. 24, 116.

[Kachelriess and Semikoz(2006)] Kachelriess, M., Semikoz, D., 2006. Astropart. Phys. 26, 10.

[Kahler et al.(2005)] Kahler, S., et al., 2005. Proc. 29th Int. Cosmic Ray Conf., Pune 10, 367.

[Kalashev et al.(2002)] Kalashev, O., et al., 2002. Phys. Rev. D 66, 063004.

[Kalges et al.(2007)] Kalges, H., et al., 2007. Proc. 30th Int. Cosmic Ray Conf., Merida, in press.

[Kalmykov et al.(1997)] Kalmykov, N., et al., 1997. Nucl. Phys. B (Proc. Suppl.) 52B, 17.

[Kampert(2007)] Kampert, K.-H., 2007. Nucl. Phys. B (Proc. Suppl.) 165, 294.

[Kampert(2008)] Kampert, K.-H., 2008. arXiv:0801.1986

[Kestel et al.(2004)] Kestel, M., et al., 2004. Nucl. Instr. \& Meth. A 535, 139.

[Klinkhammer and Risse(2008)] Klinkhammer, F., Risse, M., 2008. Phys. Rev. D $77,016002$.

[Knurenko et al.(2001)] Knurenko, S., et al., 2001. Proc. 27th Int. Cosmic Ray Conf., Hamburg 1, 177.

[Körding et al.(2008)] Körding, E., et al., 2008. Mon. Not. R. Astron. Soc. 383, 277. 
[Kronberg(1994)] Kronberg, P., 1994. Reports on Progress in Physics, 325.

[Kuz'min and Rubakov(1998)] Kuz'min, V., Rubakov, V., 1998. Phys. Atom. Nucl. 61, 1028.

[Lipari(2006)] Lipari, P., 2006. Nucl. Instr. \& Meth. A 567, 405.

[LOFAR(2007)] LOFAR, 2007. www.lofar.org.

[Lyutikov and Ouyed(1999)] Lyutikov, M., Ouyed, R., 1999. Astropart. Phys. 27, 473.

[Matthews(2005)] Matthews, J., 2005. Astropart. Phys. 22, 387.

[Medina Tanco and Watson(2001)] Medina Tanco, G., Watson, A., 2001. Proc. 27th Int. Cosmic Ray Conf., Hamburg 2, 531.

[Mollerarch et al.(2007)] Mollerarch, S., et al., 2007. Proc. 30th Int. Cosmic Ray Conf., Merida, in press (arXiv:0706.1749).

[Nagano and Watson(2000)] Nagano, M., Watson, A., 2000. Rev. Mod. Phys. 72, 689.

[Nagano et al.(1984a)] Nagano, M., et al., 1984a. J. Phys. G: Nucl. Part. Phys. 10, 1295.

[Nagano et al.(1984b)] Nagano, M., et al., 1984b. J. Phys. G: Nucl. Part. Phys. 18, 423.

[National Research Council(2003)] National Research Council, 2003. Connecting quarks with the cosmic - Eleven science questions for the new century. National Academy Press, Washington, D.C.

[Navarra et al.(2004)] Navarra, G., et al., 2004. Nucl. Instr. \& Meth. A 518, 207.

[Nitz et al.(2007)] Nitz, D., et al., 2007. Proc. 30th Int. Cosmic Ray Conf., Merida, in press (arXiv:0706.3940).

[Olinto(2000)] Olinto, A., 2000. Phys. Rep. 333, 329.

[Ong(1998)] Ong, R., 1998. Phys. Rep. 305, 93.

[Ong(2005)] Ong, R., 2005. Proc. 29th Int. Cosmic Ray Conf., Pune 10, 329.

[Ostapchenko(2005)] Ostapchenko, S., 2005. astro-ph/0412591

[Ostrowski(1999)] Ostrowski, M., 1999. Astron. \& Astroph., 134.

[Ostrowski(2002)] Ostrowski, M., 2002. Astropart. Phys. 18, 229.

[Privitera et al.(2007)] Privitera, P., et al., 2007. Proc. 30th Int. Cosmic Ray Conf., Merida, in press.

[Rachen and Biermann(1993)] Rachen, J., Biermann, P., 1993. Astron. \& Astroph. 272, 161.

[Rand and Kulkarni(1989)] Rand, R., Kulkarni, S., 1989. Astrophys. J. 343, 760.

[Ranft(1995)] Ranft, J., 1995. Phys. Rev. D 51, 64.

[Risse et al.(2005)] Risse, M., et al., 2005. Phys. Rev. Lett. 95, 171102.

[Romero et al.(1996)] Romero, G., et al., 1996. Astropart. Phys. 5, 279.

[Rubtsov et al.(2006)] Rubtsov, G., et al., 2006. Phys. Rev. D 73, 063009.

[Ryan(2005)] Ryan, J., 2005. Proc. 29th Int. Cosmic Ray Conf., Pune 10, 357. 
[Shinozaki et al.(2002)] Shinozaki, K., et al., 2002. Astrophys. J. 571, L117.

[Spiering(2003)] Spiering, C., 2003. J. Phys. G: Nucl. Part. Phys. 29, 843.

[Stanev(1997)] Stanev, T., 1997. Astrophys. J. 479, 290.

[Strong et al.(2007)] Strong, A., et al., 2007. Ann. Rev. Nucl. Part. Sci. 57, 285.

[Takeda et al.(1998)] Takeda, M., et al., 1998. Phys. Rev. Lett. 81, 1163.

[Takeda et al.(1999)] Takeda, M., et al., 1999. Astrophys. J. 522, 225.

[Takeda et al.(2003)] Takeda, M., et al., 2003. Astropart. Phys. 19, 447.

[Tinyakov and Tkachev(2001)] Tinyakov, P., Tkachev, I., 2001 74, 445.

[Tinyakov and Tkachev(2002)] Tinyakov, P., Tkachev, I., 2002. Astropart. Phys. 18, 165.

[Unger et al.(2007)] Unger, M., et al., 2007. Proc. 30th Int. Cosmic Ray Conf., Merida.

[Vallée(2004)] Vallée, J., 2004. New Astronomy Reviews 48, 763.

[van den Berg et al.(2007)] van den Berg, A., et al., 2007. Proc. 30th Int. Cosmic Ray Conf., Merida, in press.

[Véron-Cetty and Véron(2006)] Véron-Cetty, M.-P., Véron, P., 2006. Astron. \& Astroph. 455, 773 .

[Watson(2000)] Watson, A., 2000. Phys. Rep. 333 - 334, 309.

[Yamamoto et al.(2007)] Yamamoto, T., et al., 2007. Proc. 30th Int. Cosmic Ray Conf., Merida, in press, arXiv:0707.2638

[Zatsepin and Kuz'min(1966)] Zatsepin, G., Kuz'min, V., 1966. JETP Lett. 4, 78. 